\title{
SURFACE DIFFUSION OF GRAPHS: VARIATIONAL FORMULATION, ERROR ANALYSIS, AND SIMULATION*
}

\author{
EBERHARD BÄNSCH ${ }^{\dagger}$, PEDRO MORIN ${ }^{\ddagger}$, AND RICARDO H. NOCHETTO $§$
}

\begin{abstract}
Surface diffusion is a (fourth-order highly nonlinear) geometric driven motion of a surface with normal velocity proportional to the surface Laplacian of mean curvature. We present a novel variational formulation for graphs and derive a priori error estimates for a time-continuous finite element discretization. We also introduce a semi-implicit time discretization and a Schur complement approach to solve the resulting fully discrete, linear systems. After computational verification of the orders of convergence for polynomial degrees 1 and 2, we show several simulations in one dimension and two dimensions with and without forcing which explore the smoothing effect of surface diffusion, as well as the onset of singularities in finite time, such as infinite slopes and cracks.
\end{abstract}

Key words. surface diffusion, fourth-order parabolic problem, finite elements, a priori error estimates, Schur complement, smoothing effect

AMS subject classifications. 35K55, 65M12, 65M15, 65M60, 65Z05

DOI. $10.1137 / \mathrm{S} 0036142902419272$

1. Introduction. Controlling morphological changes in stressed epitaxial films is of paramount importance in materials science. The film may be thought of as subjected to mechanical stresses to model its misfit with the crystalline structure of the substrate. This in turn causes a plastic deformation of the free surface of the film, a morphological instability of the free surface which may eventually lead to crack formation and fracture. The simplest model couples surface diffusion of the free surface with linear elasticity in the bulk $[1,6,13,14,15,18,19,20]$. Investigating this complicated nonlinear dynamics requires effective and reliable computational tools.

This paper studies the geometric motion law of surface diffusion with given forcing but without elasticity. The dynamics of the free surface $\Gamma(t)$ is thus governed by the (highly nonlinear) fourth-order geometric PDE

$$
V=-\Delta_{S}(\kappa+f)
$$

where $V$ is the normal velocity of $\Gamma(t), \kappa$ is its mean curvature, and $\Delta_{S}$ is the LaplaceBeltrami operator on $\Gamma(t)$. In this reduced model $f$ is given, whereas in the full model $f$ corresponds to the elastic energy density of the bulk $\Omega(t)$ restricted to $\Gamma(t)$. Our goal is to present novel variational formulations and finite element methods for (1.1), which may be viewed as building blocks towards solving the fully coupled system.

* Received by the editors December 10, 2002; accepted for publication (in revised form) October 20, 2003; published electronically June 4, 2004. This research was partially supported by the international cooperation NSF-DAAD grants INT-9910086 and INT-0129243.

http://www.siam.org/journals/sinum/42-2/41927.html

${ }^{\dagger}$ Weierstrass Institute for Applied Analysis and Stochastics, Mohrenstrasse 39, D-10117 Berlin, Germany and Freie Universität Berlin, Arnimallee 2-6, 14195 Berlin, Germany (baensch@wiasberlin.de).

‡Departamento de Matemática, Universidad Nacional del Litoral, Instituto de Matemática Aplicada del Litoral (IMAL), Güemes 3450, 3000 Santa Fe, Argentina (pmorin@math.unl.edu.ar). The research of this author was partially supported by Programa FOMEC de la Universidad Nacional del Litoral and CONICET of Argentina and NSF grant DMS-9971450. This work was partly developed while this author was visiting the University of Maryland.

$\S$ Department of Mathematics and Institute for Physical Science and Technology, University of Maryland, College Park, MD 20742 (rhn@math.umd.edu). The research of this author was partially supported by NSF grants DMS-9971450 and DMS-0204670. 
We study the graph case in this paper and the parametric case in [3]. From now on we assume that $\Omega \subseteq \mathbb{R}^{d}(d \geq 1)$ is a fixed domain and $\Gamma(t):=\{(x, u(t, x)) \mid x \in$ $\Omega\} \subseteq \mathbb{R}^{d+1}$ is the free surface for $0 \leq t \leq T$ described by the unknown function $u$. If $Q=Q(u)=\sqrt{1+|\nabla u|^{2}}$ denotes the elementary surface area, then the unit normal $\nu$ to $\Gamma(t)$, its mean curvature $\kappa$, and the normal velocity $V$ of $\Gamma(t)$ can be expressed as follows:

$$
\nu=\frac{1}{Q}(-\nabla u, 1)^{T}, \quad \kappa=\nabla \cdot\left(\frac{\nabla u}{Q}\right), \quad V=\frac{\partial_{t} u}{Q} .
$$

Therefore, (1.1) can be written as the following system of second-order nonlinear PDEs:

$$
\frac{\partial_{t} u}{Q}=-\Delta_{S}(\kappa+f), \quad \kappa=\nabla \cdot\left(\frac{\nabla u}{Q}\right)
$$

for $(u, \kappa)$. Once completed with initial and boundary conditions, this system constitutes our starting point. Issues about existence, uniqueness, and regularity are not yet settled, not even for the graph formulation; we refer the reader to [12] for local existence for closed surfaces, as well as global existence and exponential asymptotic behavior for solutions close to a sphere. It is known, however, that the graph property may be lost in finite time [11], an intriguing situation corroborated by simulations in section 7.3.

We introduce in section 2 a new variational formulation with several crucial stability properties. Using $C^{0}$ finite elements of any degree $k \geq 1$, we obtain a space discretization in section 3 with solutions $\left(u_{h}, \kappa_{h}\right)$ and show corresponding stability properties. After deriving a number of auxiliary results for the semidiscrete scheme in section 4 , we use them to prove the quasi-optimal estimate in section 5 for the errors $e_{u}=u-u_{h}$ and $e_{\kappa}=\kappa-\kappa_{h}$ :

$$
\sup _{t \in[0, T]}\left(\left\|e_{u}\right\|_{L^{2}(\Omega)}^{2}+\int_{\Gamma_{h}(t)}\left|\nabla_{S} e_{u}\right|^{2}\right)+\int_{0}^{T}\left(\left\|e_{\kappa}\right\|_{L^{2}(\Omega)}^{2}+\int_{\Gamma_{h}(t)}\left|\nabla_{S} e_{\kappa}\right|^{2}\right) \leq C h^{2 k} .
$$

Here $C>0$ depends on the regularity of $u$ and $\kappa, k \geq 1$ is the polynomial degree, and $h$ is the mesh size. It is worth comparing our results with the existing literature. A space-time finite element method for axially symmetric surfaces is presented by Coleman, Falk, and Moakher in [7], along with several stability properties and very interesting dynamics, some not predicted by linearized stability. More recently, Deckelnick, Dziuk, and Elliott provided an error analysis [9] for the axially symmetric case. Our formulation, discretization, and analysis differ from those in [7, 9].

In section 6 we introduce a semi-implicit time discretization in the spirit of Deckelnick and Dziuk [8] and Dziuk [10]. This leads to a sequence of surfaces $\Gamma^{n}$ and linear elliptic PDEs on them. We derive again several crucial stability properties and discuss a Schur complement approach for doing effective numerical linear algebra. Finally, we show a number of numerical experiments in section 7 . Their purpose is twofold: first, we computationally verify the rate (1.3) for $k=1,2$, and, second, we explore the nonlinear regime of (1.1) via simulation. In fact, we examine the regularizing effect of surface diffusion, as well as whether (1.1) is capable of forming singularities. They manifest themselves as vertical slopes $|\nabla u|=\infty$ for $f=0$ and cracks for $f \neq 0$ of a special form. We display results for both $d=1,2$ computed with the finite element toolbox ALBERT [17, 16]. 
2. Variational formulation. In this section we write (1.2) in weak form. We start with some notation and basic formulas.

2.1. Elementary differential geometry. Let $v, w: \Omega \rightarrow \mathbb{R}$ be (smooth) functions. Since the area element is given by $Q$, then

$$
\int_{\Gamma} v=\int_{\Omega} v Q
$$

in particular, the area $A(t)$ of $\Gamma(t)$ reads $A(t)=\int_{\Omega} Q$ at time $t$. If $\tilde{v}$ is the trivial extension of $v$ to $\mathbb{R}^{d+1}$, namely, $\tilde{v}\left(x_{1}, \ldots, x_{d+1}\right):=v\left(x_{1}, \ldots, x_{d}\right)$, then the tangential gradient $\nabla_{S}$ is given by

$$
\nabla_{S} v=\nabla_{d+1} \tilde{v}-\nabla_{d+1} \tilde{v} \cdot \nu \nu
$$

where $\nabla_{d+1}$ denotes the gradient in $\mathbb{R}^{d+1}$. Since $\nabla_{d+1} \tilde{v}=\left(\nabla v^{T}, 0\right)^{T}$, we readily get

$$
\nabla_{S} v \cdot \nabla_{S} w=\nabla v \cdot \nabla w-\frac{1}{Q^{2}} \nabla v \cdot \nabla u \nabla w \cdot \nabla u
$$

Note that there is also an intrinsic definition of $\nabla_{S}$. If $\gamma=\partial \Gamma$ indicates the boundary of $\Gamma$, then this expression, together with integration by parts, yields

$$
\begin{aligned}
-\int_{\Gamma} \Delta_{S} v w+\int_{\gamma} \underline{\partial}_{\nu_{\gamma}} v w & =\int_{\Gamma} \nabla_{S} v \cdot \nabla_{S} w=\int_{\Omega} \nabla_{S} v \cdot \nabla_{S} w Q \\
& =\int_{\Omega}\left(\nabla v \cdot \nabla w Q-\frac{\nabla v \cdot \nabla u \nabla w \cdot \nabla u}{Q}\right) .
\end{aligned}
$$

Here $\nu_{\gamma}$ denotes the intrinsic outer unit normal of $\Gamma$ at $\gamma$, given by $\nu_{\gamma}=\nu_{\Gamma} \wedge \tau_{\gamma}$ with $\tau_{\gamma}$ the tangential unit vector of $\gamma$ with the appropriate sign for $\Gamma \subseteq \mathbb{R}^{3}$.

2.2. Boundary conditions and function spaces. Let $L^{p}(\Omega), 1 \leq p \leq \infty$, be the usual space of Lebesgue measurable functions with norm $\|v\|_{p}:=\left(\int_{\Omega}|v|^{p}\right)^{1 / p}$. By $\langle\cdot, \cdot\rangle$ we denote the $L^{2}$ inner product $\langle v, w\rangle:=\int_{\Omega} v w$ for $v, w \in L^{2}(\Omega)$. We indicate with $H^{m, p}(\Omega)$ the Sobolev space of functions in $L^{p}(\Omega)$ with $m$ th weak derivatives also in $L^{p}(\Omega)$ equipped with the norm $\|v\|_{m, p}:=\left(\sum_{|\alpha| \leq m} \int_{\Omega}\left|\partial_{\alpha} v\right|^{p}\right)^{1 / p}$ and $H^{m}:=H^{m, 2}$. Furthermore, $\stackrel{\circ}{H}^{1}(\Omega)^{p}$ is the subspace of functions in $H^{1, p}$ with vanishing boundary values in the sense of traces.

Finally, for a time interval $[0, T]$ and a function space $V$ we define the parabolic spaces $L^{p}(V)$ of $V$-valued functions that are measurable in time with $\|v\|_{L^{p}(V)}:=$ $\left(\int_{0}^{T}\|v(t)\|_{V}^{p} d t\right)^{1 / p}<\infty$.

To simplify the notation we will write $\|v\|_{\infty}=\|v\|_{L^{\infty}\left(L^{\infty}\right)}$. This ambiguity of notation will not lead to confusion.

We now discuss boundary conditions and corresponding function spaces $\mathcal{X}$.

Periodic boundary condition. Let $\Omega=\Pi_{i=1}^{d}\left(0, X_{i}\right)$ be a parallelogram. If $u(t, x+$ $\left.X_{i} e_{i}\right)=u(t, x), \kappa\left(t, x+X_{i} e_{i}\right)=\kappa(t, x)$ for all $x \in \partial \Omega$ and $1 \leq i \leq d$, then

$$
\mathcal{X}:=\left\{v \in H^{1}(\Omega) \mid v\left(x+X_{i} e_{i}\right)=v(x) \text { for } x \in \partial \Omega, 1 \leq i \leq d\right\} .
$$

Neumann boundary condition. If $\nu_{\gamma} \cdot \nabla_{S} u(t, x)=\nu_{\gamma} \cdot \nabla_{S} \kappa(t, x)=0$ for $x \in \partial \Omega$, then $\mathcal{X}:=H^{1}(\Omega)$.

Dirichlet boundary condition. If $u(t, x)=\kappa(t, x)=0$ for $x \in \partial \Omega$, then $\mathcal{X}:=\stackrel{\circ}{H}^{1}(\Omega)$. 
2.3. Weak form. We are now in a position to introduce two bilinear forms in $(v, w)$ and state the variational formulation of (1.2). Let

$$
\begin{aligned}
\boldsymbol{a}(u ; v, w) & :=\int_{\Omega}\left(\nabla v \cdot \nabla w Q-\frac{\nabla v \cdot \nabla u \nabla w \cdot \nabla u}{Q}\right), \\
\tilde{\boldsymbol{a}}(u ; v, w) & :=\int_{\Omega} \frac{\nabla v \cdot \nabla w}{Q} .
\end{aligned}
$$

Lemma 2.1 (equivalence). Let $u \in C^{1}\left([0, T] ; C^{4}(\bar{\Omega})\right)$, let $\kappa \in C^{0}\left([0, T] ; C^{2}(\bar{\Omega})\right)$, and let $\mathcal{X}$ be as defined in section 2.2. Then $(u, \kappa)$ is a solution of $(1.2)$ with initial value $u_{0}$ and boundary conditions as in section 2.2 iff $u(t), \kappa(t) \in \mathcal{X}$ for all $t \in[0, T]$, $u(0, \cdot)=u_{0}$, and

$$
\begin{aligned}
\left\langle\partial_{t} u, \psi\right\rangle-\boldsymbol{a}(u ; \kappa, \psi) & =\boldsymbol{a}(u ; f, \psi) & & \forall \psi \in \mathcal{X}, \\
\langle\kappa, \varphi\rangle+\tilde{\boldsymbol{a}}(u ; u, \varphi) & =0 & & \forall \varphi \in \mathcal{X} .
\end{aligned}
$$

Proof. Multiply the first equation in (1.2) by $\psi$, integrate over $\Gamma$, and use formula (2.1). Observe that the boundary term vanishes because of the choice of function space $\mathcal{X}$. Equation (2.5) follows similarly from the second equation in (1.2) integrating by parts over $\Omega$.

Remark 2.2 (mean curvature flow). In contrast to the mean curvature flow, for which a divergence formulation reads $[8,10]$

$$
\int_{\Omega} \frac{\partial_{t} u v}{Q}+\tilde{\boldsymbol{a}}(u ; u, v)=0 \quad \forall v \in \mathcal{X}
$$

we do not have the factor $\frac{1}{Q}$ in the parabolic term.

Remark 2.3 (comparing $\boldsymbol{a}$ and $\tilde{\boldsymbol{a}}$ ). The forms $\boldsymbol{a}$ and $\tilde{\boldsymbol{a}}$ are symmetric and nonnegative. If $d=1$, they coincide; i.e., $\boldsymbol{a}(u ; \cdot, \cdot)=\tilde{\boldsymbol{a}}(u ; \cdot, \cdot)$. If $d>1$, instead,

$$
\boldsymbol{a}(u ; v, u)=\tilde{\boldsymbol{a}}(u ; v, u) \quad \forall v \in \mathcal{X}
$$

because $Q\left(1-\frac{|\nabla u|^{2}}{Q^{2}}\right)=\frac{1}{Q}$. Similarly,

$$
\boldsymbol{a}(u ; v, v)=\int_{\Gamma} \nabla_{S} v \cdot \nabla_{S} v=\int_{\Omega}\left(|\nabla v|^{2} Q-\frac{|\nabla v \cdot \nabla u|^{2}}{Q}\right) \geq \int_{\Omega} \frac{|\nabla v|^{2}}{Q}=\tilde{\boldsymbol{a}}(u ; v, v) .
$$

Remark 2.4 (equivalent forms of $\boldsymbol{a}$ ). Let $\zeta:=\frac{\nabla u}{|\nabla u|}$ be a unit vector in the direction of $\nabla u$, provided that $\nabla u \neq 0$, and be arbitrary otherwise. Let $\left(\chi_{i}\right)_{i=1}^{d-1}$ be a complementary orthonormal set perpendicular to $\zeta$. A simple calculation then yields

$$
\boldsymbol{a}(u ; v, w)=\int_{\Omega}\left(\frac{\nabla v \cdot \zeta \nabla w \cdot \zeta}{Q}+Q \sum_{i=1}^{d-1} \nabla v \cdot \chi_{i} \nabla w \cdot \chi_{i}\right) \quad \forall v, w \in \mathcal{X} .
$$

Another equivalent form is obtained using $\otimes$ to denote the tensor product in $\mathbb{R}^{d}$ :

$$
\boldsymbol{a}(u ; v, w)=\int_{\Omega} \nabla v^{T}\left(Q I-\frac{\nabla u \otimes \nabla u}{Q}\right) \nabla w
$$

here $I$ denotes the identity matrix in $\mathbb{R}^{d}$. 
Remark 2.5 (volume conservation and area decrease). If the function $v=1 \in \mathcal{X}$, then (2.4) yields $0=\left\langle\partial_{t} u, 1\right\rangle=\frac{d}{d t} \int_{\Omega} u$, which is the formula for conservation of volume. On the other hand, if the forcing term $f \equiv 0$, then the area of $\Gamma(t)$ is decreasing regardless of boundary conditions (see Lemma 2.6). Both of these properties will also hold true for the semidiscrete and fully discrete formulations of section 3 and section 6 .

With the help of the above variational form of the equations, we are in a position to prove a stability result for the continuous solution.

Lemma 2.6 (continuous stability). Let $(u, \kappa)$ be a solution of (1.2) fulfilling the assumptions of Lemma 2.1, and let $A(t)$ denote the area of $\Gamma(t)$. There are two constants, $C_{1}=C_{1}(\Omega)$ and $C_{2}=C_{2}\left(\|\nabla f\|_{\infty}, A(0)\right)$, such that

$$
\begin{gathered}
\sup _{t \in[0, T]}\|u(t)\|_{2}^{2}+\int_{0}^{T}\|\kappa\|_{2}^{2} \leq\|u(0)\|_{2}^{2}+C_{1} \int_{0}^{T}\|\nabla f\|_{2}, \\
\sup _{t \in[0, T]} A(t)+\int_{0}^{T} \boldsymbol{a}(u ; \kappa, \kappa) \leq C_{2} .
\end{gathered}
$$

Moreover, if $f \equiv 0$, then the function $A(t)$ is decreasing (strictly provided that $\left.\Delta_{S} \kappa \not \equiv 0\right)$.

Proof. We omit the proof because it is the same as that of Proposition 3.2.

3. Space discretization. Let $\left(\mathcal{T}_{h}\right)_{h>0}$ be a family of (possibly graded) shape regular triangulations of $\Omega$, with $h$ being the largest size of elements in $\mathcal{T}_{h}$. We fix $k \in \mathbb{N}$ and denote by $\mathcal{X}_{h} \subseteq \mathcal{X}$ the subspace of continuous finite elements of polynomial degree $k$ with appropriate boundary conditions. Let $I_{h}: \mathcal{X} \cap C^{0}(\bar{\Omega}) \rightarrow \mathcal{X}_{h}$ be an interpolation operator fulfilling

$$
\left\|I_{h} v-v\right\|_{p}+h\left\|\nabla\left(I_{h}-v\right)\right\|_{p} \leq C h^{k+1}\|v\|_{k+1, p}
$$

for $1 \leq p \leq \infty$ and $v \in H^{k+1, p}(\Omega)[5]$. We will not need an inverse estimate for the error analysis and thus do not require quasi uniformity of the underlying meshes.

Definition 3.1 (semidiscrete solution). A pair $u_{h}, \kappa_{h}$ with $u_{h} \in C^{1}\left([0, T], \mathcal{X}_{h}\right)$, $\kappa_{h} \in C^{0}\left([0, T] ; \mathcal{X}_{h}\right)$ is called a semidiscrete solution of $(1.2)$ if $u_{h}(0, \cdot)=I_{h} u_{0}$ and

$$
\begin{aligned}
\left\langle\partial_{t} u_{h}, \psi_{h}\right\rangle-\boldsymbol{a}\left(u_{h} ; \kappa_{h}, \psi_{h}\right) & =\boldsymbol{a}\left(u_{h} ; f, \psi_{h}\right) & & \forall \psi_{h} \in \mathcal{X}_{h}, \\
\left\langle\kappa_{h}, \varphi_{h}\right\rangle+\tilde{\boldsymbol{a}}\left(u_{h} ; u_{h}, \varphi_{h}\right) & =0 & & \forall \varphi_{h} \in \mathcal{X}_{h} .
\end{aligned}
$$

From now on we consider $d \geq 2$; the analysis for $d=1$ is just a simplified version of this case. We recall from Remark 2.4 that $\left\{\zeta, \chi_{1}, \ldots, \chi_{d-1}\right\}$ is a set of orthonormal vectors for which (2.6) holds. If $\left\{\zeta_{h}, \chi_{h, 1}, \ldots, \chi_{h, d-1}\right\}$ denotes likewise a semidiscrete orthonormal set and $Q_{h}=Q\left(u_{h}\right)$, then

$$
\boldsymbol{a}\left(u_{h} ; v, w\right)=\int_{\Omega}\left(\frac{\nabla v \cdot \zeta_{h} \nabla w \cdot \zeta_{h}}{Q_{h}}+\sum_{i=1}^{d-1} \nabla v \cdot \chi_{h, i} \nabla w \cdot \chi_{h, i} Q_{h}\right) .
$$

Proposition 3.2 (semidiscrete stability). Let $\left(u_{h}, \kappa_{h}\right)$ be a semidiscrete solution in the sense of Definition 3.1, and let $A_{h}(t):=\int_{\Omega} Q_{h}$ denote the area of the surface $\Gamma_{h}(t):=\left\{\left(x, u_{h}(t, x)\right) \mid x \in \Omega\right\}$. There are two constants, $C_{1}=C_{1}(\Omega)$ and 
$C_{2}=C_{2}\left(\|\nabla f\|_{\infty}, A_{h}(0)\right)$, such that

$$
\begin{gathered}
\sup _{t \in[0, T]}\left\|u_{h}(t)\right\|_{2}^{2}+\int_{0}^{T}\left\|\kappa_{h}\right\|_{2}^{2} \leq\left\|u_{h}(0)\right\|_{2}^{2}+C_{1} \int_{0}^{T}\|\nabla f\|_{2}, \\
\sup _{t \in[0, T]} A_{h}(t)+\int_{0}^{T} \boldsymbol{a}\left(u_{h} ; \kappa_{h}, \kappa_{h}\right) \leq C_{2} .
\end{gathered}
$$

Moreover, if $f \equiv 0$, the function $A_{h}(t)$ is decreasing (strictly if $\boldsymbol{a}\left(u_{h} ; \kappa_{h}, \kappa_{h}\right)>0$ ).

Proof. First, choose $\psi_{h}:=u_{h}, \varphi_{h}:=\kappa_{h}$ as test functions in (3.2) and (3.3), respectively. In view of Remark 2.3 , we get

$$
\left\langle\partial_{t} u_{h}, u_{h}\right\rangle+\left\langle\kappa_{h}, \kappa_{h}\right\rangle+\underbrace{\tilde{\boldsymbol{a}}\left(u_{h} ; u_{h}, \kappa_{h}\right)-\boldsymbol{a}\left(u_{h} ; \kappa_{h}, u_{h}\right)}_{=0}=\boldsymbol{a}\left(u_{h} ; f, u_{h}\right),
$$

and, since $\left|\nabla u_{h}\right| / Q_{h} \leq 1$,

$$
\boldsymbol{a}\left(u_{h} ; f, u_{h}\right)=\tilde{\boldsymbol{a}}\left(u_{h} ; f, u_{h}\right)=\int_{\Omega} \frac{\nabla f \cdot \nabla u_{h}}{Q_{h}} \leq\|\nabla f\|_{2}\left(\int_{\Omega} \frac{\left|\nabla u_{h}\right|^{2}}{Q_{h}^{2}}\right)^{1 / 2} \leq C_{1}\|\nabla f\|_{2} .
$$

Integrating in time gives (3.5). We next set $\psi_{h}:=-\kappa_{h}, \varphi_{h}:=\partial_{t} u_{h}$ to derive

$$
\underbrace{-\left\langle\partial_{t} u_{h}, \kappa_{h}\right\rangle+\left\langle\kappa_{h}, \partial_{t} u_{h}\right\rangle}_{=0}+\boldsymbol{a}\left(u_{h} ; \kappa_{h}, \kappa_{h}\right)+\tilde{\boldsymbol{a}}\left(u_{h} ; u_{h}, \partial_{t} u_{h}\right)=-\boldsymbol{a}\left(u_{h} ; f, \kappa_{h}\right) .
$$

Observing that

$$
\tilde{\boldsymbol{a}}\left(u_{h} ; u_{h}, \partial_{t} u_{h}\right)=\int_{\Omega} \frac{\nabla u_{h} \cdot \nabla \partial_{t} u_{h}}{Q_{h}}=\frac{d}{d t} \int_{\Omega} Q_{h}=\frac{d}{d t} A_{h}(t),
$$

we get

$$
\frac{d}{d t} A_{h}(t)+\boldsymbol{a}\left(u_{h} ; \kappa_{h}, \kappa_{h}\right)=-\boldsymbol{a}\left(u_{h} ; f, \kappa_{h}\right),
$$

which implies that $A_{h}(t)$ is decreasing, provided that $f \equiv 0$. To prove (3.6) for $f \not \equiv 0$, we have to bound $\boldsymbol{a}\left(u_{h} ; f, \kappa_{h}\right)$. Making use of (3.4), we obtain

$$
\begin{aligned}
\boldsymbol{a}\left(u_{h} ; f, \kappa_{h}\right) & =\int_{\Omega}\left(\frac{\nabla f \cdot \zeta_{h} \nabla \kappa_{h} \cdot \zeta_{h}}{Q_{h}}+\sum_{i=1}^{d-1} \nabla f \cdot \chi_{h, i} \nabla \kappa_{h} \cdot \chi_{h, i} Q_{h}\right) \\
& \leq\|\nabla f\|_{\infty} \int_{\Omega}\left(\frac{\left|\nabla \kappa_{h} \cdot \zeta_{h}\right|}{Q_{h}}+\sum_{i=1}^{d-1}\left|\nabla \kappa_{h} \cdot \chi_{h, i}\right| Q_{h}\right) \\
& \leq\|\nabla f\|_{\infty}\left(\frac{|\Omega|}{4 \epsilon}+\epsilon \int_{\Omega}\left(\frac{\left|\nabla \kappa_{h} \cdot \zeta_{h}\right|^{2}}{Q_{h}}+\sum_{i=1}^{d-1}\left|\nabla \kappa_{h} \cdot \chi_{h, i}\right|^{2} Q_{h}\right)+\frac{\int_{\Omega} Q_{h}}{4 \epsilon}\right) \\
& =\|\nabla f\|_{\infty}\left(C_{\epsilon}+\epsilon \boldsymbol{a}\left(u_{h} ; \kappa_{h}, \kappa_{h}\right)+C_{\epsilon} A_{h}(t)\right),
\end{aligned}
$$

where we have used that $Q_{h} \geq 1$. Choosing $\epsilon$ sufficiently small, a Gronwall argument finally yields (3.6).

Corollary 3.3 (global existence of semidiscrete solution). For $h>0$ and $T>0$ there is a unique semidiscrete solution $\left(u_{h}, \kappa_{h}\right)$ fulfilling (3.2) and (3.3). 
Proof. Observing that (3.2)-(3.3) is equivalent to a system of ODEs with a locally Lipschitz right-hand side, we get a local in time existence of the semidiscrete solution. Using the above stability estimate, this solution can be extended to the time interval $[0, T]$ by standard arguments. Uniqueness follows from the local Lipschitz continuity of the right-hand side.

4. Auxiliary estimates. In this section we present some auxiliary lemmas and results that will be instrumental in deriving the error estimates. Since they will be used several times and might be of independent interest, we present them separately.

We start by introducing the following notation:

$$
e_{u}:=u-u_{h}, \quad e_{\kappa}:=\kappa-\kappa_{h}, \quad N_{h}:=\int_{\Omega}\left|\nu-\nu_{h}\right|^{2} Q_{h}
$$

LEMma 4.1 (basic geometric formulas). Using the notation introduced above, the following inequalities hold:

$$
\left|\frac{1}{Q}-\frac{1}{Q_{h}}\right| \leq\left|\nu-\nu_{h}\right|, \quad\left|Q-Q_{h}\right| \leq Q Q_{h}\left|\nu-\nu_{h}\right|,
$$

and

$$
\left|\frac{\nabla u \otimes \nabla u}{Q}-\frac{\nabla u_{h} \otimes \nabla u_{h}}{Q_{h}}\right| \leq 3 Q Q_{h}\left|\nu-\nu_{h}\right|
$$

Proof. Recalling that $\nu=\frac{1}{Q}(\nabla u,-1)^{T}$ and $\nu_{h}=\frac{1}{Q_{h}}\left(\nabla u_{h},-1\right)^{T}$, the inequalities in (4.1) are immediate. To prove (4.2), let us introduce the notation $z:=\frac{\nabla u}{Q}$ and $z_{h}:=\frac{\nabla u_{h}}{Q_{h}}$, and observe that

$$
\begin{aligned}
\frac{\nabla u \otimes \nabla u}{Q}-\frac{\nabla u_{h} \otimes \nabla u_{h}}{Q_{h}} & =z \otimes z Q-z_{h} \otimes z_{h} Q_{h} \\
& =\left(z-z_{h}\right) \otimes z Q+z_{h} \otimes z\left(Q-Q_{h}\right)+z_{h} \otimes\left(z-z_{h}\right) Q_{h} .
\end{aligned}
$$

Therefore, the triangle inequality and the fact that $\left|z-z_{h}\right| \leq\left|\nu-\nu_{h}\right|$ yield (4.2).

The following lemma is crucial for our error analysis and provides a coercivity estimate for $\tilde{\boldsymbol{a}}$. The estimate is the same as the one that appears in the error analysis for mean curvature flow and is due to Deckelnick and Dziuk [8] and Dziuk [10]. Even though its proof can be found in [8, p. 347], we sketch it here for completeness.

Lemma 4.2 (coercivity of $\tilde{\boldsymbol{a}}$ ). The following estimate holds true:

$$
\tilde{\boldsymbol{a}}\left(u ; u, \partial_{t} e_{u}\right)-\tilde{\boldsymbol{a}}\left(u_{h} ; u_{h}, \partial_{t} e_{u}\right) \geq \frac{1}{2} \frac{d}{d t} N_{h}(t)-\left\|\nabla \partial_{t} u(t)\right\|_{\infty} N_{h}(t) .
$$

Proof. We start with two geometric relations which follow by simple calculation:

$$
1-\frac{1+\nabla u \cdot \nabla u_{h}}{Q Q_{h}}=\frac{1}{2}\left|\nu-\nu_{h}\right|^{2}, \quad\left|\left(\frac{1}{Q}-\frac{1}{Q_{h}}\right)\left(\frac{\nabla u}{Q}-\frac{\nabla u_{h}}{Q_{h}}\right)\right| \leq \frac{1}{2}\left|\nu-\nu_{h}\right|^{2}
$$


We now use the first equality in (4.3) to realize that

$$
\begin{aligned}
\frac{1}{2} \partial_{t}\left(\left|\nu-\nu_{h}\right|^{2} Q_{h}\right)= & \partial_{t}\left(\left(1-\frac{1+\nabla u \cdot \nabla u_{h}}{Q Q_{h}}\right) Q_{h}\right) \\
= & \frac{\nabla u_{h} \cdot \nabla \partial_{t} u_{h}}{Q_{h}}+\frac{\nabla u \cdot \nabla \partial_{t} u}{Q^{3}}\left(1+\nabla u \cdot \nabla u_{h}\right) \\
& -\frac{1}{Q}\left(\nabla u_{h} \cdot \nabla \partial_{t} u+\nabla u \cdot \nabla \partial_{t} u_{h}\right),
\end{aligned}
$$

and, upon adding and subtracting $\frac{\nabla u_{h} \cdot \partial_{t} \nabla u}{Q_{h}}$ and reordering terms, we find out that

$$
\begin{aligned}
\frac{1}{2} \partial_{t}\left(\left|\nu-\nu_{h}\right|^{2} Q_{h}\right)= & \left(\frac{\nabla u}{Q}-\frac{\nabla u_{h}}{Q_{h}}\right) \cdot \nabla \partial_{t}\left(u-u_{h}\right) \\
& -\partial_{t} \nabla u \cdot\left(\frac{\nabla u}{Q}-\frac{\nabla u_{h}}{Q_{h}}+\frac{\nabla u_{h}}{Q}-\frac{1+\nabla u \cdot \nabla u_{h}}{Q^{2}} \frac{\nabla u}{Q}\right) .
\end{aligned}
$$

We next integrate over $\Omega$, use the definition of $N_{h}$, and add and subtract $\nabla \partial_{t} u \cdot \nabla u \frac{Q_{h}}{Q^{2}}$ to obtain

$$
\begin{aligned}
\tilde{\boldsymbol{a}}\left(u ; u, \partial_{t} e_{u}\right)-\tilde{\boldsymbol{a}}\left(u_{h} ; u_{h}, \partial_{t} e_{u}\right)= & \int_{\Omega}\left(\frac{\nabla u}{Q}-\frac{\nabla u_{h}}{Q_{h}}\right) \cdot \nabla \partial_{t}\left(u-u_{h}\right) \\
= & \frac{1}{2} \frac{d}{d t} \int_{\Omega}\left|\nu-\nu_{h}\right|^{2} Q_{h} \\
& +\int_{\Omega} \partial_{t} \nabla u \cdot\left(\frac{\nabla u}{Q}-\frac{\nabla u_{h}}{Q_{h}}\right)\left(\frac{1}{Q_{h}}-\frac{1}{Q}\right) Q_{h} \\
& +\int_{\Omega} \partial_{t} \nabla u \cdot \frac{\nabla u}{Q^{2}}\left(1-\frac{1+\nabla u \cdot \nabla u_{h}}{Q Q_{h}}\right) Q_{h} \\
\geq & \frac{1}{2} \frac{d}{d t} N_{h}(t)-\left\|\nabla \partial_{t} u(t)\right\|_{\infty} N_{h}(t),
\end{aligned}
$$

where we have employed both estimates (4.3). This finally concludes the proof.

The following two lemmas are consistency estimates for the bilinear forms $\boldsymbol{a}$ and $\tilde{\boldsymbol{a}}$, respectively.

Lemma 4.3 (consistency estimate for $\boldsymbol{a}$ ). For every $\epsilon>0$ there exists a constant $C=C\left(\epsilon,\|\nabla \kappa\|_{\infty},\|Q\|_{\infty},\|\nabla f\|_{\infty}\right)>0$ such that

$$
\left|\boldsymbol{a}(u ; \kappa, w)-\boldsymbol{a}\left(u_{h} ; \kappa_{h}, w\right)\right| \leq \epsilon \boldsymbol{a}\left(u_{h} ; e_{\kappa}, e_{\kappa}\right)+C\|\nabla w\|_{\infty}^{2}+N_{h}(t) \quad \forall w \in \mathcal{X} .
$$

Proof. We first add and subtract the term $\boldsymbol{a}\left(u_{h} ; \kappa, w\right)$ to obtain $\boldsymbol{a}(u ; \kappa, w)-\boldsymbol{a}\left(u_{h} ; \kappa_{h}, w\right)=\boldsymbol{a}\left(u_{h} ; \kappa-\kappa_{h}, w\right)+\left(\boldsymbol{a}(u ; \kappa, w)-\boldsymbol{a}\left(u_{h} ; \kappa, w\right)\right)=:(I)+(I I)$ and analyze $(I)$ and $(I I)$ separately. By the Cauchy-Schwarz inequality,

$$
(I) \leq \epsilon \boldsymbol{a}\left(u_{h} ; e_{\kappa}, e_{\kappa}\right)+\frac{1}{4 \epsilon} \boldsymbol{a}\left(u_{h} ; w, w\right),
$$

and, using the definition $(2.2)$ of $\boldsymbol{a}\left(u_{h} ; \cdot, \cdot\right)$, we get

$$
\boldsymbol{a}\left(u_{h} ; w, w\right)=\int_{\Omega}|\nabla w|^{2} Q_{h}-\frac{1}{Q_{h}}\left|\nabla w \cdot \nabla u_{h}\right|^{2} \leq \int_{\Omega}|\nabla w|^{2} Q_{h} \leq\|\nabla w\|_{\infty}^{2} A_{h}(t) .
$$


Therefore,

$$
(I) \leq \epsilon \boldsymbol{a}\left(u_{h} ; e_{\kappa}, e_{\kappa}\right)+\frac{1}{4 \epsilon}\|\nabla w\|_{\infty}^{2} A_{h}(t) .
$$

We now turn to estimate (II). Using the equivalent form (2.7) for $\boldsymbol{a}$, we have

$$
(I I)=\int_{\Omega} \nabla \kappa^{T}\left(\left(Q-Q_{h}\right) I-\left(\frac{\nabla u \otimes \nabla u}{Q}-\frac{\nabla u_{h} \otimes \nabla u_{h}}{Q_{h}}\right)\right) \nabla w .
$$

By (4.1) and (4.2), the integrand in (II) is bounded by $4 Q Q_{h}|\nabla \kappa||\nabla w|\left|\nu-\nu_{h}\right|$, which by the Cauchy-Schwarz inequality gives

$$
\begin{aligned}
(I I) & \leq 4 \int_{\Omega} Q^{2}|\nabla \kappa|^{2}|\nabla w|^{2} Q_{h}+\int_{\Omega}\left|\nu-\nu_{h}\right|^{2} Q_{h} \\
& \leq 4\|Q\|_{\infty}^{2}\|\nabla \kappa\|_{\infty}^{2}\|\nabla w\|_{\infty}^{2} A_{h}(t)+N_{h}(t) .
\end{aligned}
$$

Since $A_{h}(t) \leq C$ from (3.6), the bounds for $(I)$ and $(I I)$ yield the assertion.

Lemma 4.4 (consistency estimate for $\tilde{\boldsymbol{a}}$ ). For every $\epsilon>0$ we have

$$
\left|\tilde{\boldsymbol{a}}(u ; u, w)-\tilde{\boldsymbol{a}}\left(u_{h} ; u_{h}, w\right)\right| \leq \epsilon \tilde{\boldsymbol{a}}\left(u_{h} ; w, w\right)+\frac{1}{4 \epsilon} N_{h}(t) \quad \forall w \in \mathcal{X} .
$$

Proof. Using the definition (2.3) of $\tilde{\boldsymbol{a}}$ and the Cauchy-Schwarz inequality, we get

$$
\begin{aligned}
\left|\tilde{\boldsymbol{a}}(u ; u, w)-\tilde{\boldsymbol{a}}\left(u_{h} ; u_{h}, w\right)\right| & \leq \int_{\Omega}\left|\frac{\nabla u}{Q}-\frac{\nabla u_{h}}{Q_{h}}\right||\nabla w| \leq \int_{\Omega}\left|\nu-\nu_{h}\right||\nabla w| \\
& \leq \epsilon \int_{\Omega} \frac{|\nabla w|^{2}}{Q_{h}}+\frac{1}{4 \epsilon} N_{h}(t)=\epsilon \tilde{\boldsymbol{a}}\left(u_{h} ; w, w\right)+\frac{1}{4 \epsilon} N_{h}(t),
\end{aligned}
$$

which is the desired estimate.

The following lemma establishes another consistency estimate for $\boldsymbol{a}$, this time provided that solely the nonlinear part of $\boldsymbol{a}$ changes.

Lemma 4.5. There exists a constant $C=C\left(\|Q\|_{\infty}\right)>0$ such that for every $\epsilon>0$

$$
\left|\boldsymbol{a}(u ; v, w)-\boldsymbol{a}\left(u_{h} ; v, w\right)\right| \leq \epsilon \boldsymbol{a}\left(u_{h} ; w, w\right)+\frac{C}{\epsilon}\|\nabla v\|_{\infty}^{2} N_{h}(t) \quad \forall v, w \in \mathcal{X} .
$$

Proof. With $R:=\|Q\|_{\infty}$, we consider the following disjoint splitting of $\Omega: \Omega=$ $\Omega^{+} \cup \Omega^{-}$with $\Omega^{+}:=\left\{x \in \Omega \mid Q_{h}(x)>2 R\right\}$ and $\Omega^{-}:=\left\{x \in \Omega \mid Q_{h}(x) \leq 2 R\right\}$.

We first estimate the integrand of $\boldsymbol{a}(u ; \cdot, \cdot)-\boldsymbol{a}\left(u_{h} ; \cdot, \cdot\right)$ in the case $x \in \Omega^{-}$. According to (2.7), we consider this integrand written in the form

$$
\nabla v^{T}\left(\left(Q-Q_{h}\right) I-\left(\frac{\nabla u \otimes \nabla u}{Q}-\frac{\nabla u_{h} \otimes \nabla u_{h}}{Q_{h}}\right)\right) \nabla w=:(I) .
$$

Since $Q_{h}(x) \leq 2 R$ for $x \in \Omega^{-}$, in view of (4.1) and (4.2) we have

$$
\begin{aligned}
(I) & \leq 4|\nabla v||\nabla w| Q Q_{h}\left|\nu-\nu_{h}\right| \leq 8 R^{2}|\nabla v| \frac{|\nabla w|}{\sqrt{Q_{h}}}\left|\nu-\nu_{h}\right| \sqrt{Q_{h}} \\
& \leq \epsilon \frac{|\nabla w|^{2}}{Q_{h}}+16 \frac{R^{4}}{\epsilon}\|\nabla v\|_{\infty}^{2}\left|\nu-\nu_{h}\right|^{2} Q_{h} .
\end{aligned}
$$


To analyze the case $x \in \Omega^{+}$, we choose $\zeta, \zeta_{h}, \chi_{i}, \chi_{h, i}$ as in Remark 2.4. Since $Q(x) \leq R$ and $Q_{h}(x)>2 R$ we have

$$
\left|\nu-\nu_{h}\right| \geq \frac{1}{Q}-\frac{1}{Q_{h}} \geq \frac{1}{2 R} \quad \text { and } \quad\left|\zeta-\zeta_{h}\right|,\left|\chi_{i}-\chi_{h, i}\right| \leq 2 \leq 4 R\left|\nu-\nu_{h}\right| .
$$

Consider the integrand in the form (2.6):

$$
\underbrace{\left(\frac{\nabla v \cdot \zeta \nabla w \cdot \zeta}{Q}-\frac{\nabla v \cdot \zeta_{h} \nabla w \cdot \zeta_{h}}{Q_{h}}\right)}_{(I I)}+\sum_{i=1}^{d-1} \underbrace{\nabla w^{T}\left(\chi_{i} \otimes \chi_{i} Q-\chi_{h, i} \otimes \chi_{h, i} Q_{h}\right) \nabla v}_{(I I I)_{i}} .
$$

Since $R \geq 1$, we have for $(I I)$

$$
\begin{aligned}
(I I) & =\nabla v^{T}\left(\frac{\zeta \otimes \zeta}{Q}-\frac{\zeta_{h} \otimes \zeta_{h}}{Q_{h}}\right) \nabla w \\
& =\nabla v^{T}\left[\left(\zeta-\zeta_{h}\right) \otimes \zeta \frac{1}{Q}+\zeta_{h} \otimes \zeta\left(\frac{1}{Q}-\frac{1}{Q_{h}}\right)+\zeta_{h} \otimes\left(\zeta-\zeta_{h}\right) \frac{1}{Q_{h}}\right] \nabla w \\
& \leq C R|\nabla v||\nabla w|\left|\nu-\nu_{h}\right| \leq \frac{C^{2} R^{2}}{4 \epsilon}\|\nabla v\|_{\infty}^{2}\left|\nu-\nu_{h}\right|^{2} Q_{h}+\epsilon \frac{|\nabla w|^{2}}{Q_{h}} .
\end{aligned}
$$

For $(I I I)_{i}$, instead, we proceed as follows with the aid of (4.1):

$$
\begin{aligned}
(I I I)_{i} & =\nabla w^{T}\left(\left(\chi_{i}-\chi_{h, i}\right) \otimes \chi_{i} Q+\chi_{h, i} \otimes \chi_{i}\left(Q-Q_{h}\right)+\chi_{h, i} \otimes\left(\chi_{i}-\chi_{h, i}\right) Q_{h}\right) \nabla v \\
& \leq 4 R^{2}\left|\nu-\nu_{h}\right||\nabla v||\nabla w|+5 R\left|\nu-\nu_{h}\right| Q_{h}\left|\chi_{h, i} \cdot \nabla w\right||\nabla v| \\
& \leq \epsilon \frac{|\nabla w|^{2}}{Q_{h}}+\epsilon\left|\nabla w \cdot \chi_{h, i}\right|^{2} Q_{h}+\frac{C R^{4}}{\epsilon}\|\nabla v\|_{\infty}^{2}\left|\nu-\nu_{h}\right|^{2} Q_{h} .
\end{aligned}
$$

Collecting the estimates for both cases, $x \in \Omega^{-}$and $x \in \Omega^{+}$, integrating over $\Omega$, and recalling (2.6), we obtain the assertion after relabeling $\epsilon$.

Using Lemma 4.5 we obtain a coercivity estimate for $\boldsymbol{a}$.

Corollary 4.6 (coercivity of $\boldsymbol{a}$ ). There exists $C=C\left(\|Q\|_{\infty}\right)>0$ such that

$$
\boldsymbol{a}\left(u ; \kappa, e_{\kappa}\right)-\boldsymbol{a}\left(u_{h} ; \kappa_{h}, e_{\kappa}\right) \geq \frac{1}{2} \boldsymbol{a}\left(u_{h} ; e_{\kappa}, e_{\kappa}\right)-C\|\nabla \kappa\|_{\infty}^{2} N_{h}(t)
$$

Proof. Adding and subtracting $\boldsymbol{a}\left(u_{h} ; \kappa, e_{\kappa}\right)$, and using Lemma 4.5 with $\epsilon=1 / 2$, we readily obtain the desired estimate.

LEMma 4.7 (coercivity of $\left.N_{h}(t)\right)$. There exists $C=C\left(\|Q\|_{\infty}\right)$ such that

$$
\boldsymbol{a}\left(u_{h} ; e_{u}, e_{u}\right) \leq C N_{h}(t) .
$$

Proof. In light of Remark 2.4, we can write

$$
\boldsymbol{a}\left(u_{h} ; e_{u}, e_{u}\right)=\int_{\Omega} \underbrace{\frac{\left|\nabla e_{u} \cdot \zeta_{h}\right|^{2}}{Q_{h}}}_{(I)}+\sum_{i=1}^{d-1} \int_{\Omega} \underbrace{\left|\nabla e_{u} \cdot \chi_{h, i}\right|^{2} Q_{h}}_{(I I)_{i}} .
$$

By virtue of (4.1), (I) satisfies

$$
\begin{aligned}
(I) & \leq \frac{\left|\nabla e_{u}\right|^{2}}{Q_{h}}=\frac{\left|\nabla u-\nabla u_{h}\right|^{2}}{Q_{h}} \leq \frac{\left|\nu Q-\nu_{h} Q_{h}\right|^{2}}{Q_{h}} \\
& \leq \frac{\left|\nu\left(Q-Q_{h}\right)+\left(\nu-\nu_{h}\right) Q_{h}\right|^{2}}{} Q_{h} \leq 4\|Q\|_{\infty}^{2} Q_{h}\left|\nu-\nu_{h}\right|^{2} .
\end{aligned}
$$


To treat the integrand $(I I)_{i}$ we again split $\Omega$ into $\Omega^{-}$and $\Omega^{+}$, as in Lemma 4.5. Consider first $x \in \Omega^{-}$, namely, $Q_{h}(x) \leq 2 R$ with $R:=\|Q(t)\|_{\infty}$. As in (4.5), we get

$$
\left|\nabla e_{u} \cdot \chi_{h, i}\right|^{2} Q_{h} \leq 4 R^{2} \frac{\left|\nabla e_{u}\right|^{2}}{Q_{h}} \leq 16\|Q\|_{\infty}^{4} Q_{h}\left|\nu-\nu_{h}\right|^{2} .
$$

Now we consider $Q_{h}(x)>2 R$. Since $\nabla u_{h} \cdot \chi_{h, i}=0$, it follows from (4.4) that

$$
\nabla e_{u} \cdot \chi_{h, i}=\nabla\left(u-u_{h}\right) \cdot \chi_{h, i}=\nabla u \cdot \chi_{h, i} \leq|\nabla u| \leq 2 R\|\nabla u\|_{\infty}\left|\nu-\nu_{h}\right|,
$$

whence

$$
\left|\nabla e_{u} \cdot \chi_{h, i}\right|^{2} Q_{h} \leq 4\|Q\|_{\infty}^{4}\left|\nu-\nu_{h}\right|^{2} Q_{h} .
$$

The desired estimate then follows by integration over $\Omega$.

5. A priori error analysis. In this section we prove the main theoretical result of this article, which can be stated as follows.

TheOREM 5.1. Let $\left(u_{h}, \kappa_{h}\right)$ be the semidiscrete solution of Definition 3.1, and let $e_{u}:=u-u_{h}, e_{\kappa}:=\kappa-\kappa_{h}$. There exists a constant $C$ depending on $\|\nabla f\|_{\infty}$, $\left\|\partial_{t} u\right\|_{L^{2}\left(H^{k+1}(\Omega)\right)},\left\|\partial_{t} \nabla u\right\|_{\infty},\|\kappa\|_{L^{2}\left(H^{k+1}(\Omega)\right)},\left\|\partial_{t} \kappa\right\|_{L^{2}\left(H^{k}(\Omega)\right)}$, and $\|\nabla \kappa\|_{\infty}$ such that

$$
\sup _{t \in[0, T]}\left(\left\|e_{u}(t)\right\|_{2}^{2}+\int_{\Gamma_{h}(t)}\left|\nabla_{S} e_{u}\right|^{2}\right)+\int_{0}^{T}\left(\left\|e_{\kappa}\right\|_{2}^{2}+\int_{\Gamma_{h}(t)}\left|\nabla_{S} e_{\kappa}\right|^{2}\right) \leq C h^{2 k} .
$$

The proof of Theorem 5.1 is a consequence of two estimates, the strong and the weak estimates, derived from the error equations (5.1) and (5.2) below by choosing appropriate test functions.

Remark $5.2\left(H^{1}\right.$ estimate). The estimate for $\nabla_{S} e_{u}$ might seem surprising at first sight since direct $H^{1}$ estimates are unavailable for minimal surfaces for dimension $d>2$. It is thus worth stressing that, instead of the usual $H^{1}$ norm, we have an integral over the discrete surface $\Gamma_{h}(t)$ which involves the tangential gradient $\nabla_{S}$. In its derivation, we exploit parabolicity to prove first an estimate for $N_{h}(t)$ and then use Lemma 4.7 (see section 5.1).

Remark 5.3 (regularity). The required regularity of $(u, \kappa)$ in Theorem 5.1 might appear inconsistent with that of $\Omega$ for polynomial degree $k>1$. In fact, we have assumed that $\Omega$ can be partitioned exactly into finite elements but not that $\Omega$ is polyhedral (see section 3). This would thus entail the use of isoparametric elements for $k>1$, but still $\Omega=\Omega_{h}$ might fail to hold. Accounting for the effect of $\Omega \neq \Omega_{h}$ is mostly a technical issue and is, therefore, omitted in the subsequent discussion, which is already rather technical.

5.1. Proof of Theorem 5.1. Subtracting (3.2) and (3.3) from (2.4) and (2.5), respectively, we get the following error equations:

$$
\begin{aligned}
\left\langle\partial_{t} e_{u}, \psi_{h}\right\rangle-\left(\boldsymbol{a}\left(u ; \kappa, \psi_{h}\right)-\boldsymbol{a}\left(u_{h} ; \kappa_{h}, \psi_{h}\right)\right) & =\boldsymbol{a}\left(u ; f, \psi_{h}\right)-\boldsymbol{a}\left(u_{h} ; f, \psi_{h}\right), \\
\left\langle e_{\kappa}, \varphi_{h}\right\rangle+\left(\tilde{\boldsymbol{a}}\left(u ; u, \varphi_{h}\right)-\tilde{\boldsymbol{a}}\left(u_{h} ; u_{h}, \varphi_{h}\right)\right) & =0
\end{aligned}
$$

for all $\psi_{h}, \varphi_{h} \in \mathcal{X}_{h}$. The strong and weak estimates below are formulated in terms of the following interpolation errors, which can be bounded via (3.1):

$$
\rho_{u}:=u-I_{h} u, \quad \rho_{\kappa}:=\kappa-I_{h} \kappa_{h} .
$$


The strong estimate of section 5.2 reads as follows: For all $\epsilon>0$ there exists a constant $C_{0}$ depending only on $\|\nabla f\|_{\infty},\|\nabla \kappa\|_{\infty},\left\|\partial_{t} \nabla u\right\|_{\infty}$, and $\epsilon$ such that for $t \in[0, T]$

$$
\begin{aligned}
N_{h}(t)+ & \int_{0}^{t} \boldsymbol{a}\left(u_{h} ; e_{\kappa}, e_{\kappa}\right) \leq N_{h}(0)+C_{0} \int_{0}^{t}\left(N_{h}+\left\|e_{u}\right\|_{2}^{2}\right) \\
& +2 \epsilon\left(\left\|e_{u}(t)\right\|_{2}^{2}+\int_{0}^{t}\left\|e_{\kappa}\right\|_{2}^{2}\right) \\
& +\frac{1}{2 \epsilon}\left\|\rho_{\kappa}(t)\right\|_{2}^{2}+\left\|e_{u}(0)\right\|_{2}^{2}+\left\|\rho_{\kappa}(0)\right\|_{2}^{2} \\
& +C_{0} \int_{0}^{t}\left(\left\|\nabla \rho_{\kappa}\right\|_{\infty}^{2}+\left\|\partial_{t} \nabla \rho_{u}\right\|_{2}^{2}+\left\|\partial_{t} \rho_{u}\right\|_{2}^{2}+\left\|\partial_{t} \rho_{\kappa}\right\|_{2}^{2}\right) .
\end{aligned}
$$

It is clear that to close the argument we need separate control on the term multiplied by $\epsilon$ of the right-hand side of (5.4). This is provided by the weak estimate of section 5.3 , which reads as follows: There exist constants $C_{1}, C_{2}$ depending on $\|\nabla f\|_{\infty}$ and $\|Q\|_{\infty}$ such that for $t \in[0, T]$ we have

$$
\begin{aligned}
\frac{1}{2}\left\|e_{u}(t)\right\|_{2}^{2}+\int_{0}^{t}\left\|e_{\kappa}\right\|_{2}^{2} \leq & \frac{1}{2}\left\|e_{u}(0)\right\|_{2}^{2}+\int_{0}^{t}\left\|e_{u}\right\|_{2}^{2} \\
& +C_{1} \int_{0}^{t} N_{h}+\frac{1}{2} \int_{0}^{t} \boldsymbol{a}\left(u_{h} ; e_{\kappa}, e_{\kappa}\right) \\
& +\int_{0}^{t}\left(\left\|\nabla \rho_{\kappa}\right\|_{2}^{2}+\left\|\rho_{\kappa}\right\|_{2}^{2}+\left\|\partial_{t} \rho_{u}\right\|_{2}^{2}\right) \\
& +2\left\|\rho_{u}(t)\right\|_{2}^{2}+C_{2} \int_{0}^{t}\left\|\nabla \rho_{u}\right\|_{\infty}^{2} .
\end{aligned}
$$

To prove Theorem 5.1 we add (5.4) and (5.5) and then choose $\epsilon=1 / 8$ to eliminate $\left\|e_{u}(t)\right\|_{2}^{2}+\int_{0}^{t}\left\|e_{\kappa}\right\|_{2}^{2}$ from the right-hand side. Employing a Gronwall argument, we can also remove $\int_{0}^{t}\left(N_{h}(s)+\left\|e_{u}\right\|_{2}^{2}\right)$ from the right-hand side at the expense of an exponential depending on $C_{0}, C_{1}$, and $T$. Finally, Lemma 4.7, in conjunction with $\boldsymbol{a}\left(u_{h} ; v, v\right)=\int_{\Gamma_{h}} \nabla_{S} v \cdot \nabla_{S} v$, yields the left-hand side of the asserted estimate. Its right-hand side and underlying a priori regularity result from applying (3.1) to the terms involving $\rho_{\kappa}, \rho_{u}$ defined in (3.1).

5.2. Strong estimate (5.4). To prove (5.4), we choose the discrete functions

$$
\begin{aligned}
-\psi_{h} & :=I_{h} \kappa-\kappa_{h}=\left(\kappa-\kappa_{h}\right)+\left(I_{h} \kappa-\kappa\right)=e_{\kappa}-\rho_{\kappa} \in \mathcal{X}_{h}, \\
\varphi_{h} & :=\partial_{t}\left(I_{h} u-u_{h}\right)=\partial_{t} e_{u}-\partial_{t} \rho_{u} \in \mathcal{X}_{h} .
\end{aligned}
$$

Adding (5.1) and (5.2), and invoking Lemma 4.2 and Corollary 4.6, we get

$$
\begin{aligned}
\frac{1}{2} \frac{d}{d t} N_{h}(t)+ & \frac{1}{2} \boldsymbol{a}\left(u_{h} ; e_{\kappa}, e_{\kappa}\right)-C N_{h}(t) \\
\leq & \boldsymbol{a}\left(u ; \kappa, e_{\kappa}\right)-\boldsymbol{a}\left(u_{h} ; \kappa_{h}, e_{\kappa}\right)+\tilde{\boldsymbol{a}}\left(u ; u, \partial_{t} e_{u}\right)-\tilde{\boldsymbol{a}}\left(u_{h} ; u_{h}, \partial_{t} e_{u}\right) \\
= & \left(\boldsymbol{a}\left(u ; \kappa, \rho_{\kappa}\right)-\boldsymbol{a}\left(u_{h} ; \kappa_{h}, \rho_{\kappa}\right)\right)-\left(\boldsymbol{a}\left(u ; f, e_{\kappa}-\rho_{\kappa}\right)-\boldsymbol{a}\left(u_{h} ; f, e_{\kappa}-\rho_{\kappa}\right)\right) \\
& +\left(\tilde{\boldsymbol{a}}\left(u ; u, \partial_{t} \rho_{u}\right)-\tilde{\boldsymbol{a}}\left(u_{h} ; u_{h}, \partial_{t} \rho_{u}\right)\right)-\left\langle\partial_{t} e_{u}, \rho_{\kappa}\right\rangle+\left\langle e_{\kappa}, \partial_{t} \rho_{u}\right\rangle \\
= & :(I)+(I I)+(I I I)+(I V)+(V),
\end{aligned}
$$


with $C$ depending only on $\left\|\partial_{t} \nabla u\right\|_{\infty},\|\nabla \kappa\|_{\infty}$, and $\|Q\|_{\infty}$. We now proceed to estimate each term on the right-hand side separately.

By Lemma 4.3 , there is a constant $C=C\left(\epsilon,\|\nabla \kappa\|_{\infty},\|Q\|_{\infty},\|\nabla f\|_{\infty}\right)$ such that

$$
|(I)| \leq \epsilon \boldsymbol{a}\left(u_{h} ; e_{\kappa}, e_{\kappa}\right)+C\left\|\nabla \rho_{\kappa}\right\|_{\infty}^{2}+N_{h}(t) .
$$

Using Lemma 4.4 with $\epsilon=1$, we obtain

$$
|(I I I)| \leq \tilde{\boldsymbol{a}}\left(u_{h} ; \partial_{t} \rho_{u}, \partial_{t} \rho_{u}\right)+N_{h}(t) \leq\left\|\nabla \partial_{t} \rho_{u}\right\|_{2}^{2}+N_{h}(t) .
$$

For any $t \in[0, T]$ we integrate $(I V)$ by parts on $[0, t]$, thereby obtaining

$$
\begin{aligned}
& \int_{0}^{t}(I V)=\left\langle e_{u}(0), \rho_{\kappa}(0)\right\rangle-\left\langle e_{u}(t), \rho_{\kappa}(t)\right\rangle+\int_{0}^{t}\left\langle e_{u}, \partial_{t} \rho_{\kappa}\right\rangle \\
& \quad \leq \frac{1}{2}\left\|e_{u}(0)\right\|_{2}^{2}+\frac{1}{2}\left\|\rho_{\kappa}(0)\right\|_{2}^{2}+\frac{\epsilon}{2}\left\|e_{u}(t)\right\|_{2}^{2}+\frac{1}{2 \epsilon}\left\|\rho_{\kappa}(t)\right\|_{2}^{2}+\frac{1}{2} \int_{0}^{t}\left(\left\|e_{u}\right\|_{2}^{2}+\left\|\partial_{t} \rho_{\kappa}\right\|_{2}^{2}\right) .
\end{aligned}
$$

For $(V)$ we readily have

$$
|(V)| \leq \frac{\epsilon}{2}\left\|e_{\kappa}\right\|_{2}^{2}+\frac{1}{2 \epsilon}\left\|\partial_{t} \rho_{u}\right\|_{2}^{2} .
$$

We decompose $(I I)$ into discretization and interpolation errors as follows:

$$
-(I I)=\underbrace{\left(\boldsymbol{a}\left(u ; f, e_{\kappa}\right)-\boldsymbol{a}\left(u_{h} ; f, e_{\kappa}\right)\right)}_{(I I)_{e}}-\underbrace{\left(\boldsymbol{a}\left(u ; f, \rho_{\kappa}\right)-\boldsymbol{a}\left(u_{h} ; f, \rho_{\kappa}\right)\right)}_{(I I)_{\rho}} .
$$

In light of Lemma 4.5 , there is a constant $C=C\left(\|Q\|_{\infty}\right)$ such that

$$
\left|(I I)_{e}\right| \leq \frac{1}{4} \boldsymbol{a}\left(u_{h} ; e_{\kappa}, e_{\kappa}\right)+C\|\nabla f\|_{\infty}^{2} N_{h}(t) .
$$

Using Lemma 4.1 and (3.6), we find a constant $C=C\left(\|\nabla f\|_{\infty},\|Q\|_{\infty}, A_{h}(0)\right)$ such that

$$
\begin{aligned}
\left|(I I)_{\rho}\right| & =\left|\int_{\Omega} \nabla f^{T}\left(\left(Q-Q_{h}\right) I-\left(\frac{\nabla u \otimes \nabla u}{Q}-\frac{\nabla u_{h} \otimes \nabla u_{h}}{Q_{h}}\right)\right) \nabla \rho_{\kappa}\right| \\
& \leq 4 \int_{\Omega}|\nabla f|\left|\nabla \rho_{\kappa}\right| Q Q_{h}\left|\nu-\nu_{h}\right| \\
& \leq 4\|\nabla f\|_{\infty}^{2}\left\|\nabla \rho_{\kappa}\right\|_{\infty}^{2}\|Q\|_{\infty}^{2} \int_{\Omega} Q_{h}+\int_{\Omega}\left|\nu-\nu_{h}\right|^{2} Q_{h} \\
& \leq C\left\|\nabla \rho_{\kappa}\right\|_{\infty}^{2} A_{h}(t)+N_{h}(t) \leq C\left\|\nabla \rho_{\kappa}\right\|_{\infty}^{2}+N_{h}(t) .
\end{aligned}
$$

Finally, collecting the above estimates for $(I)$ to $(V)$, subtracting $\frac{1}{4} \boldsymbol{a}\left(u_{h} ; e_{\kappa}, e_{\kappa}\right)$, and integrating in time from 0 to $t \in[0, T]$, we arrive at (5.4).

5.3. Weak estimate (5.5). To prove (5.5), we choose the discrete functions

$$
\begin{aligned}
\psi_{h} & :=I_{h} u-u_{h}=e_{u}-\rho_{u} \in \mathcal{X}_{h}, \\
\varphi_{h} & :=I_{h} \kappa-\kappa_{h}=e_{\kappa}-\rho_{\kappa} \in \mathcal{X}_{h} .
\end{aligned}
$$


Adding the error equations (5.1) and (5.2), we obtain

$$
\begin{aligned}
\left\langle\partial_{t} e_{u}, e_{u}\right\rangle+\left\langle e_{\kappa}, e_{\kappa}\right\rangle= & \left(\boldsymbol{a}\left(u ; \kappa, e_{u}\right)-\boldsymbol{a}\left(u_{h} ; \kappa_{h}, e_{u}\right)\right)-\left(\tilde{\boldsymbol{a}}\left(u ; u, e_{\kappa}\right)-\tilde{\boldsymbol{a}}\left(u_{h} ; u_{h}, e_{\kappa}\right)\right) \\
& +\left\langle\partial_{t} e_{u}, \rho_{u}\right\rangle-\left(\boldsymbol{a}\left(u ; \kappa, \rho_{u}\right)-\boldsymbol{a}\left(u_{h} ; \kappa_{h}, \rho_{u}\right)\right) \\
& +\left(\boldsymbol{a}\left(u ; f, e_{u}-\rho_{u}\right)-\boldsymbol{a}\left(u_{h} ; f, e_{u}-\rho_{u}\right)\right)+\left\langle e_{\kappa}, \rho_{\kappa}\right\rangle \\
& +\left(\tilde{\boldsymbol{a}}\left(u ; u, \rho_{\kappa}\right)-\tilde{\boldsymbol{a}}\left(u_{h} ; u_{h}, \rho_{\kappa}\right)\right) \\
= & :(I)+\cdots+(V I I) .
\end{aligned}
$$

We proceed now to bound each term from $(I)$ to $(V I I)$ separately.

Adding and subtracting $\boldsymbol{a}\left(u_{h} ; \kappa, e_{u}\right)$ to $(I)$, and employing Lemma 4.5 with $\epsilon=\frac{1}{6}$, we readily have

$$
\begin{aligned}
|(I)| & \leq\left|\boldsymbol{a}\left(u ; \kappa, e_{u}\right)-\boldsymbol{a}\left(u_{h} ; \kappa, e_{u}\right)\right|+\left|\boldsymbol{a}\left(u_{h} ; e_{\kappa}, e_{u}\right)\right| \\
& \leq C\|\nabla \kappa\|_{\infty}^{2} N_{h}(t)+\frac{5}{2} \boldsymbol{a}\left(u_{h} ; e_{u}, e_{u}\right)+\frac{1}{6} \boldsymbol{a}\left(u_{h} ; e_{\kappa}, e_{\kappa}\right) .
\end{aligned}
$$

Consequently, Lemma 4.7 yields the following bound with $C=C\left(\|\nabla \kappa\|_{\infty},\|Q\|_{\infty}\right)$ :

$$
|(I)| \leq C N_{h}(t)+\frac{1}{6} \boldsymbol{a}\left(u_{h} ; e_{\kappa}, e_{\kappa}\right) .
$$

Making use of Lemma 4.4 and Remark 2.3, we readily deduce (using $\epsilon=\frac{1}{6}$ )

$$
|(I I)| \leq \frac{1}{6} \tilde{\boldsymbol{a}}\left(u_{h} ; e_{\kappa}, e_{\kappa}\right)+\frac{3}{2} N_{h}(t) \leq \frac{1}{6} \boldsymbol{a}\left(u_{h} ; e_{\kappa}, e_{\kappa}\right)+\frac{3}{2} N_{h}(t),
$$

as well as (using $\epsilon=\frac{1}{2}$ )

$$
|(V I I)| \leq \frac{1}{2} \tilde{\boldsymbol{a}}\left(u_{h} ; \rho_{\kappa}, \rho_{\kappa}\right)+\frac{1}{2} N_{h}(t) \leq \frac{1}{2}\left\|\nabla \rho_{\kappa}\right\|_{2}^{2}+\frac{1}{2} N_{h}(t) .
$$

Using Lemma 4.3 with $\epsilon=\frac{1}{6}$ we find a constant $C=C\left(\|\nabla \kappa\|_{\infty},\|Q\|_{\infty},\|\nabla f\|_{\infty}\right)$ such that

$$
|(I V)| \leq \frac{1}{6} \boldsymbol{a}\left(u_{h} ; e_{\kappa}, e_{\kappa}\right)+N_{h}(t)+C\left\|\nabla \rho_{u}\right\|_{\infty}^{2} .
$$

For $(V I)$, we obviously have $|(V I)| \leq \frac{1}{2}\left\|e_{\kappa}\right\|_{2}^{2}+\frac{1}{2}\left\|\rho_{\kappa}\right\|_{2}^{2}$. For $(I I I)$, instead, we integrate by parts on $[0, t]$ for any $t \in[0, T]$ to obtain

$$
\begin{aligned}
\int_{0}^{t}(I I I) & =\left\langle e_{u}(t), \rho_{u}(t)\right\rangle-\left\langle e_{u}(0), \rho_{u}(0)\right\rangle-\int_{0}^{t}\left\langle e_{u}, \partial_{t} \rho_{u}\right\rangle \\
& \leq \frac{1}{4}\left\|e_{u}(t)\right\|_{2}^{2}+\left\|\rho_{u}(t)\right\|_{2}^{2}+\frac{1}{2} \int_{0}^{t}\left(\left\|e_{u}\right\|_{2}^{2}+\left\|\partial_{t} \rho_{u}\right\|_{2}^{2}\right) .
\end{aligned}
$$

It remains to bound $(V)$, which involves the right-hand side $f$. Applying Lemma 4.5 (with $\epsilon=1$ ) and Lemma 4.7, we obtain

$$
\left|\boldsymbol{a}\left(u ; f, e_{u}\right)-\boldsymbol{a}\left(u_{h} ; f, e_{u}\right)\right| \leq C\|\nabla f\|_{\infty}^{2} N_{h}(t)+\boldsymbol{a}\left(u_{h} ; e_{u}, e_{u}\right) \leq C N_{h}(t),
$$

with $C=C\left(\|\nabla f\|_{\infty},\|Q\|_{\infty}\right)$. Since $\boldsymbol{a}\left(u ; f, \rho_{u}\right)-\boldsymbol{a}\left(u_{h} ; f, \rho_{u}\right)$ is similar to $(I I)_{\rho}$ in (5.6), we likewise deduce

$$
\left|\boldsymbol{a}\left(u ; f, \rho_{u}\right)-\boldsymbol{a}\left(u_{h} ; f, \rho_{u}\right)\right| \leq C\left\|\nabla \rho_{u}\right\|_{\infty}^{2} A_{h}(t)+N_{h}(t) \leq C\left\|\nabla \rho_{u}\right\|_{\infty}^{2}+N_{h}(t),
$$


whence, for $C$ depending on $\|\nabla f\|_{\infty}$ and $\|Q\|_{\infty}$, we end up with

$$
|(V)| \leq C\left\|\nabla \rho_{u}\right\|_{\infty}^{2}+C N_{h}(t) .
$$

Inserting the above bounds for $(I)$ to $(V I I)$ back into (5.7) and integrating from 0 to $t$, we finally obtain the desired estimate (5.5).

6. Full discretization. In this section we introduce the fully discrete scheme actually used in simulations, along with the linear algebra approach to its solution.

6.1. Definition and properties. To discretize in time we subdivide the time interval into $t_{0}=0<t_{1}<\cdots<t_{N}=T$ and set $\tau_{n}:=t_{n+1}-t_{n}$. We define the notion of semi-implicit fully discrete problem as follows: Set $u_{h}^{0}=u_{h}(0)$ and for $n=0,1, \ldots, N-1$ determine $u_{h}^{n+1}, \kappa_{h}^{n+1} \in \mathcal{X}_{h}$ by

$$
\begin{aligned}
\left\langle u_{h}^{n+1}, \psi_{h}\right\rangle-\tau_{n} \boldsymbol{a}\left(u_{h}^{n} ; \kappa_{h}^{n+1}, \psi_{h}\right) & =\tau_{n} \boldsymbol{a}\left(u_{h}^{n} ; f^{n}, \psi_{h}\right)+\left\langle u_{h}^{n}, \psi_{h}\right\rangle & & \forall \psi_{h} \in \mathcal{X}_{h}, \\
\left\langle\kappa_{h}^{n+1}, \varphi_{h}\right\rangle+\tilde{\boldsymbol{a}}\left(u_{h}^{n} ; u_{h}^{n+1}, \varphi_{h}\right) & =0 & & \forall \varphi_{h} \in \mathcal{X}_{h},
\end{aligned}
$$

with $f^{n}:=f\left(t_{n}\right)$. Existence and uniqueness of solutions $u_{h}^{n}, \kappa_{h}^{n}$ follow from the considerations in section 6.2.

We now establish a stability estimate analogous to (3.6) in Lemma 2.6.

THEOREM 6.1 (fully discrete stability). Let $\left(u_{h}^{n}, \kappa_{h}^{n}\right)_{n=0}^{N}$ be a solution of the fully discrete equations (6.1) and (6.2), and let $A_{h}^{n}:=\int_{\Omega} Q\left(u_{h}^{n}\right)$ denote the area of the surface $\Gamma_{h}^{n}:=\left\{\left(x, u_{h}^{n}(x)\right) \mid x \in \Omega\right\}$. There exists $C=C\left(\|\nabla f\|_{\infty}, A_{h}^{0}\right)$ such that

$$
\sup _{1 \leq n \leq N} A_{h}^{n}+\sum_{n=1}^{N} \tau_{n} \int_{\Gamma_{h}^{n-1}}\left|\nabla_{S} \kappa_{h}^{n}\right|^{2} \leq C .
$$

Moreover, if $f \equiv 0, A_{h}^{n}$ is a decreasing sequence (strictly if $\boldsymbol{a}\left(u_{h}^{n-1} ; \kappa_{h}^{n}, \kappa_{h}^{n}\right)>0$ ).

Proof. Choose as test functions $-\kappa_{h}^{n+1}$ and $\left(u_{h}^{n+1}-u_{h}^{n}\right)$ in (6.1) and (6.2), respectively, and add both equations. One readily gets

$$
\tau_{n} \boldsymbol{a}\left(u_{h}^{n} ; \kappa_{h}^{n+1}, \kappa_{h}^{n+1}\right)+\int_{\Omega} \frac{\nabla u_{h}^{n+1} \cdot \nabla\left(u_{h}^{n+1}-u_{h}^{n}\right)}{Q\left(u_{h}^{n}\right)}=-\tau_{n} \boldsymbol{a}\left(u_{h}^{n} ; f^{n}, \kappa_{h}^{n+1}\right) .
$$

The next step consists of finding a discrete counterpart of (3.7). Observing that

$$
|a|-|b| \leq \frac{a \cdot(a-b)}{|b|} \quad \forall a, b \in \mathbb{R}^{d+1}
$$

and setting $a:=\left(\nabla u_{h}^{n+1}, 1\right)^{T}, b:=\left(\nabla u_{h}^{n}, 1\right)^{T}$, we obtain

$$
Q\left(u_{h}^{n+1}\right)-Q\left(u_{h}^{n}\right) \leq \frac{\nabla u_{h}^{n+1} \cdot \nabla\left(u_{h}^{n+1}-u_{h}^{n}\right)}{Q\left(u_{h}^{n}\right)} .
$$

Inserting this into (6.4) gives $A_{h}^{n+1} \leq A_{h}^{n}$ if $f \equiv 0$. To prove (6.3) for $f \not \equiv 0$, we have to bound the right-hand side in (6.4). This can be done similarly to (3.5), obtaining

$$
\left|\boldsymbol{a}\left(u_{h}^{n} ; f^{n}, \kappa_{h}^{n+1}\right)\right| \leq C\left(1+A_{h}^{n}\right)+\epsilon \boldsymbol{a}\left(u_{h}^{n} ; \kappa_{h}^{n+1}, \kappa_{h}^{n+1}\right),
$$

with $C=C\left(\epsilon,\|\nabla f\|_{\infty}\right)$. Multiplying by $\tau_{n}$, choosing $\epsilon$ sufficiently small, summing up over all $n$, and using a discrete Gronwall argument, the result follows. 
6.2. Schur complement strategy. Let $\mathcal{X}_{h}=\operatorname{span}\left\{\varphi_{j}\right\} \subseteq \mathcal{X}$ with the usual nodal basis functions $\varphi_{j}$ and the corresponding nodal space $\underline{\mathbf{X}}$. Then, for the time instant $t_{n+1}$, the fully discrete system of equations can be rewritten as

$$
\left[\begin{array}{cc}
\tilde{A} & M \\
M^{T} & -\tau_{n} A
\end{array}\right]\left[\underline{\underline{U}}^{n+1} \underline{\underline{K}}^{n+1}\right]=\left[\begin{array}{c}
0 \\
M^{T} \underline{U}^{n}+\tau_{n} \underline{F}^{n}
\end{array}\right]
$$

where $\underline{U}^{n}, \underline{K}^{n}$ denote the vector of nodal values for $u_{h}^{n}, \kappa_{h}^{n}$, respectively,

$$
u_{h}^{n}=\sum_{j} \underline{U}_{j}^{n} \varphi_{j}, \quad \kappa_{h}^{n}=\sum_{j} \underline{K}_{j}^{n} \varphi_{j},
$$

the vector $\underline{F}^{n}$ is given by $\underline{F}_{j}^{n}=\boldsymbol{a}\left(u_{h}^{n} ; f^{n}, \varphi_{j}\right)$, and the matrices $M, A, \tilde{A}$ are given by

$$
M_{i, j}=\left\langle\varphi_{j}, \varphi_{i}\right\rangle, \quad A_{i, j}=\boldsymbol{a}\left(u_{h}^{n} ; \varphi_{j}, \varphi_{i}\right), \quad \tilde{A}_{i, j}=\tilde{\boldsymbol{a}}\left(u_{h}^{n} ; \varphi_{j}, \varphi_{i}\right) .
$$

Notice that the matrices $A$ and $\tilde{A}$ depend on $u_{h}^{n}$ and thus have to be reassembled in every time step.

To derive a Schur complement formulation, we have to distinguish between the various boundary conditions (see section 2.2 ).

Dirichlet boundary conditions. In this case, since $\mathcal{X}:=\stackrel{\circ}{H}^{1}(\Omega)$, the matrix $\tilde{A}$ is invertible, and a Schur complement for $\underline{K}^{n+1}$ is thus given by

$$
\begin{aligned}
\left(M^{T} \tilde{A}^{-1} M+\tau_{n} A\right) \underline{K}^{n+1} & =-M^{T} \underline{U}^{n}-\tau_{n} \underline{F}^{n}, \\
\tilde{A} \underline{U}^{n+1} & =-M \underline{K}^{n+1} .
\end{aligned}
$$

This system is decoupled and uniquely solvable for both $\underline{K}^{n+1}$ and $\underline{U}^{n+1}$.

Periodic and Neumann boundary conditions. This case is a bit more involved because constant functions are in $\mathcal{X}_{h}$, whence $\tilde{A}$ has a kernel $\operatorname{ker}(\tilde{A})=\operatorname{span}\{\underline{1}\}$.

Let $\underline{\mathbf{V}}, \underline{\mathbf{W}} \subseteq \underline{\mathbf{X}}$ be the spaces of nodal values for $\underline{U}^{n+1}$ defined by

$$
\underline{\mathbf{V}}:=\{\underline{V} \mid \underline{1} \cdot M V=0\}, \quad \underline{\mathbf{W}}:=\{\underline{V} \mid \underline{1} \cdot \underline{V}=0\}=\operatorname{span}\{\underline{1}\}^{\perp} .
$$

Multiplying the first equation in (6.5) by $\underline{1}$, we see that $\underline{1} \cdot M \underline{K}^{n+1}=0$, which means that $\underline{K}^{n+1} \in \underline{\mathbf{V}}$. Let $P$ be the orthogonal projection onto $\operatorname{span}\{\underline{1}\}$ with respect to the Euclidean inner product in $\mathbb{R}^{I}$, with $I=\operatorname{dim} \underline{\mathbf{X}}$. If $S:=\left(\tilde{A}_{\mid \underline{\mathbf{W}}}\right)^{-1}$, then

$$
S M \underline{K}^{n+1}=-S \tilde{A} \underline{U}^{n+1}=-(I d-P) \underline{U}^{n+1}=-\underline{U}^{n+1}+P \underline{U}^{n+1},
$$

or $\underline{U}^{n+1}=-S M \underline{K}^{n+1}+P \underline{U}^{n+1}$. Consequently, using the second equation in (6.5),

$$
\left(M^{T} S M+\tau_{n} A\right) \underline{K}^{n+1}-M^{T} P \underline{U}^{n+1}=-M^{T} \underline{U}^{n}-\tau_{n} \underline{F}^{n} .
$$

Now let $\Pi:=I d-\frac{M^{T} 1 \otimes M^{T} 1}{\left|M^{T} \underline{1}\right|^{2}}$ be the orthogonal projection onto $\underline{\mathbf{V}}$. Applying $\Pi$ to both sides of (6.6) and using that $\Pi M P \underline{U}^{n+1}=0$ and $\Pi \underline{K}^{n+1}=\underline{K}^{n+1}$, we arrive at

$$
\Pi\left(M^{T} S M+\tau_{n} A\right) \Pi \underline{K}^{n+1}=-\Pi\left(M^{T} \underline{U}^{n}+\tau_{n} \underline{F}^{n}\right) .
$$

The matrix $\Pi\left(M^{T} S M+\tau_{n} A\right) \Pi$ is symmetric and positive definite in $\underline{\mathbf{V}}$, and thus (6.7) is uniquely solvable for $\underline{K}^{n+1}$ in $\underline{\mathbf{V}}$. Finally, $\underline{U}^{n+1}$ is uniquely determined by

$$
\tilde{A} \underline{U}^{n+1}=-M \underline{K}^{n+1}, \quad \underline{1} \cdot M^{T} \underline{U}^{n+1}=\underline{1} \cdot M^{T} \underline{U}^{n} .
$$

Note that the last equation is the conservation of volume $\int_{\Omega}\left(U^{n+1}-U^{n}\right)=0$ written in matrix-vector form; compare with Remark 2.5. 
7. Numerical experiments. The purpose of this section is to document via several experiments the performance of the discretization scheme proposed in this article. We open this section with some comments about the implementation of the algorithm within the flexible adaptive finite element toolbox ALBERT [16, 17]. We continue with a verification of the experimental orders of convergence (EOCs) achieved by the method with different polynomial degrees and relations between time step $\tau$ and mesh size $h$. We next illustrate the smoothing effect of surface diffusion (case $f=0$ ), and we finally present simulations driven by a forcing term which exhibit singularity formation in finite time in both one dimension and two dimensions (case $f \neq 0)$.

7.1. Implementation. The matrices of section 6 were assembled using the standard assembling tools of ALBERT, and the solution to the linear systems (6.7)-(6.8) was obtained by a conjugate gradient method.

For the assembling of the linear systems, quadrature rules exact for polynomials of degree $2 k$ were used, where $k$ is the degree of the finite element. For computing the errors versus the exact solution, quadratures of order $2 k+2$ were used.

For all the experiments presented in this article, domains with periodic boundary conditions were considered. Experiments with other boundary conditions were also carried out and will be shown elsewhere. The results were similar.

7.2. EOCs. To test the performance of the discretization scheme we consider the domain $\Omega=(-1,1) \times(-1,1) \subset \mathbb{R}^{2}$ with the exact solution

$$
u(x, y, t)=1+0.1 \sin (\pi x) \sin (2 \pi y) \cos (\pi t) \quad \forall t \in[0,1] .
$$

The exact curvature $\kappa=\nabla \cdot\left(\frac{\nabla u}{Q}\right)$ and right-hand side $F=\partial_{t} u-Q \Delta_{S} \kappa$ were obtained using the symbolic capabilities of Mathematica. The finite element method of section 6 is used to compute $\left(u_{h}, \kappa_{h}\right)$, and a comparison with $(u, \kappa)$ is presented in Tables 7.17.4. They display the errors

$$
\begin{aligned}
& \operatorname{err}_{\nu}:=\sup _{0 \leq t \leq T}\left(\int_{\Omega}\left|\nu-\nu_{h}\right|^{2} Q_{h}\right)^{1 / 2}, \quad \operatorname{err}_{u}:=\sup _{0 \leq t \leq T} \boldsymbol{a}\left(u_{h} ; e_{u}, e_{u}\right)^{1 / 2}, \\
& \operatorname{err}_{\kappa}:=\left(\int_{0}^{T} \boldsymbol{a}\left(u_{h} ; e_{\kappa}, e_{\kappa}\right)\right)^{1 / 2}, \quad \operatorname{err}_{u, 2}:=\sup _{0 \leq t \leq T}\left\|e_{u}\right\|_{2}, \quad \operatorname{err}_{\kappa, 2}:=\left(\int_{0}^{T}\left\|e_{\kappa}\right\|_{2}^{2}\right)^{1 / 2}
\end{aligned}
$$

for different values of $h$ and $\tau$ along with the EOCs. Given two meshes with mesh sizes $H, h$ and errors $\operatorname{err}_{H}$, $\operatorname{err}_{h}$, respectively, the EOC is determined according to

$$
\mathrm{EOC}=\frac{\log \left(\operatorname{err}_{H} / \operatorname{err}_{h}\right)}{\log (H / h)}
$$

which gives the computational exponent $k$ in the expression $\operatorname{err}_{h} \cong C h^{k}$.

In Table 7.1 we show the results obtained using linear elements and a time step $\tau=h$. Even though $\tau$ seems to be large as compared to $h$, the convergence rate is still linear, and no instabilities arise. This is not so surprising if we recall that the fully discrete system is unconditionally stable (see Theorem 6.1). In order to verify the error analysis in section 5 for the semidiscretization in space, we also compute the EOCs for smaller values of $\tau$, namely $\tau=0.1 h$ and $\tau=h^{2}$; see Tables 7.2 and 7.3. Here again, we observe that the EOCs are at least 1. Moreover, as one would expect, the errors measured in $L^{2}(\Omega)$ norms are approximately of second order, provided 
TABLE 7.1

Linear elements and time step $\tau=h$.

\begin{tabular}{|l|cc|cc|cc|cc|cc|}
\hline \multicolumn{1}{|c|}{$h$} & $\operatorname{err}_{\nu}$ & $\mathrm{EOC}$ & $\operatorname{err}_{u}$ & $\mathrm{EOC}$ & $\operatorname{err}_{\kappa}$ & $\mathrm{EOC}$ & $\operatorname{err}_{u, 2}$ & $\mathrm{EOC}$ & $\operatorname{err}_{\kappa, 2}$ & $\mathrm{EOC}$ \\
\hline $1 / 2$ & 0.5601 & & 0.6055 & & 18.2 & & 0.0836 & & 2.1921 & \\
$1 / 4$ & 0.2549 & 1.14 & 0.2884 & 1.07 & 7.70 & 1.24 & 0.0287 & 1.54 & 0.4366 & 2.33 \\
$1 / 8$ & 0.1297 & 0.97 & 0.1448 & 0.99 & 4.66 & 0.73 & 0.0121 & 1.24 & 0.1773 & 1.30 \\
$1 / 16$ & 0.0636 & 1.03 & 0.0708 & 1.03 & 2.41 & 0.95 & 0.0049 & 1.32 & 0.0630 & 1.49 \\
$1 / 32$ & 0.0310 & 1.03 & 0.0344 & 1.04 & 1.21 & 0.99 & 0.0021 & 1.24 & 0.0262 & 1.26 \\
\hline
\end{tabular}

TABLE 7.2

Linear elements and time step $\tau=0.1 \mathrm{~h}$.

\begin{tabular}{|l|cc|cc|cc|cc|cc|}
\hline \multicolumn{1}{|c|}{$h$} & $\operatorname{err}_{\nu}$ & $\mathrm{EOC}$ & $\operatorname{err}_{u}$ & $\mathrm{EOC}$ & $\operatorname{err}_{\kappa}$ & $\mathrm{EOC}$ & $\operatorname{err}_{u, 2}$ & $\mathrm{EOC}$ & $\operatorname{err}_{\kappa, 2}$ & $\mathrm{EOC}$ \\
\hline $1 / 2$ & 0.5594 & & 0.6048 & & 18.4 & & 0.0834 & & 2.2249 & \\
$1 / 4$ & 0.2463 & 1.18 & 0.2772 & 1.13 & 7.67 & 1.26 & 0.0251 & 1.73 & 0.4071 & 2.45 \\
$1 / 8$ & 0.1240 & 0.99 & 0.1364 & 1.02 & 4.67 & 0.71 & 0.0081 & 1.62 & 0.1484 & 1.46 \\
$1 / 16$ & 0.0611 & 1.02 & 0.0669 & 1.03 & 2.40 & 0.96 & 0.0022 & 1.87 & 0.0397 & 1.90 \\
$1 / 32$ & 0.0304 & 1.01 & 0.0332 & 1.01 & 1.19 & 1.00 & 0.0006 & 1.85 & 0.0102 & 1.97 \\
\hline
\end{tabular}

TABLE 7.3

Linear elements and time step $\tau=h^{2}$.

\begin{tabular}{|l|cc|cc|cc|cc|cc|}
\hline \multicolumn{1}{|c|}{$h$} & $\operatorname{err}_{\nu}$ & $\mathrm{EOC}$ & $\operatorname{err}_{u}$ & $\mathrm{EOC}$ & $\operatorname{err}_{\kappa}$ & $\mathrm{EOC}$ & $\operatorname{err}_{u, 2}$ & $\mathrm{EOC}$ & $\operatorname{err}_{\kappa, 2}$ & $\mathrm{EOC}$ \\
\hline $1 / 2$ & 0.5597 & & 0.6051 & & 18.4 & & 0.0835 & & 2.2214 & \\
$1 / 4$ & 0.2470 & 1.18 & 0.2782 & 1.12 & 7.67 & 1.26 & 0.0254 & 1.71 & 0.4073 & 2.45 \\
$1 / 8$ & 0.1240 & 0.99 & 0.1365 & 1.03 & 4.61 & 0.73 & 0.0082 & 1.63 & 0.1466 & 1.47 \\
$1 / 16$ & 0.0611 & 1.02 & 0.0669 & 1.03 & 2.38 & 0.96 & 0.0022 & 1.93 & 0.0392 & 1.90 \\
$1 / 32$ & 0.0304 & 1.01 & 0.0332 & 1.01 & 1.19 & 1.00 & 0.0005 & 1.98 & 0.0099 & 1.99 \\
\hline
\end{tabular}

TABLE 7.4

Quadratic elements and time step $\tau=h^{2}$.

\begin{tabular}{|l|cc|cc|cc|cc|cc|}
\hline \multicolumn{1}{|c|}{$h$} & $\operatorname{err}_{\nu}$ & $\mathrm{EOC}$ & $\operatorname{err}_{u}$ & $\mathrm{EOC}$ & $\operatorname{err}_{\kappa}$ & $\mathrm{EOC}$ & $\operatorname{err}_{u, 2}$ & $\mathrm{EOC}_{\kappa, 2}$ & $\operatorname{EOC}$ \\
\hline $1 / 2$ & 0.1271 & & 0.1376 & & 7.38 & & 0.0101 & & 0.3277 & \\
$1 / 4$ & 0.0419 & 1.60 & 0.0487 & 1.50 & 2.47 & 1.58 & 0.0040 & 1.35 & 0.0797 & 2.04 \\
$1 / 8$ & 0.0102 & 2.03 & 0.0122 & 1.99 & 0.71 & 1.80 & 0.0009 & 2.19 & 0.0152 & 2.39 \\
$1 / 16$ & 0.0025 & 2.01 & 0.0030 & 2.00 & 0.17 & 2.07 & 0.0002 & 2.11 & 0.0032 & 2.24 \\
\hline
\end{tabular}

that $\tau=h^{2}$; this is not predicted by our theory though. For $\tau=h, 0.1 h$ we do not recover second-order errors because the time discretization error - expected to be of first order-dominates the space error in $L^{2}(\Omega)$ norms.

To further verify experimentally the error estimates of section 5 , which are valid for any polynomial degree, we also compute the EOCs for quadratic elements. Table 7.4 displays the results obtained with quadratics and $\tau=h^{2}$. The EOCs are about 2 in all the error norms, as predicted by theory, including those in $L^{2}(\Omega)$. In fact, the latter cannot exhibit EOCs close to 3 due to the choice of the time step $\tau=h^{2}$.

7.3. Smoothing effect in one dimension: Case $\boldsymbol{f} \equiv \mathbf{0}$. In this section we present experimental results in $\Omega=(-1,1)$ concerning the behavior of the discrete solution when $f \equiv 0$ and $u_{0}(x)=1+\delta(x)$ is a perturbation of the stationary solution $u \equiv 1$.

Superposition of sines. We consider the perturbation

$$
\delta(x)=0.1 \sin (\pi x)+0.3 \sin (16 \pi x),
$$




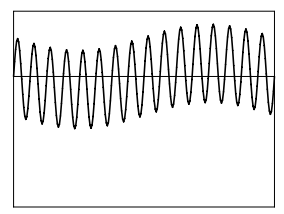

$t=0$

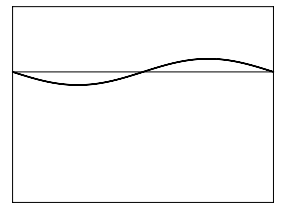

$t=1 \times 10^{-4}$

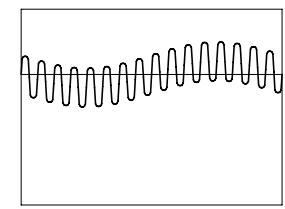

$t=1 \times 10^{-5}$

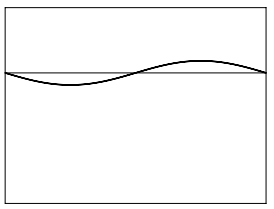

$t=1 \times 10^{-3}$

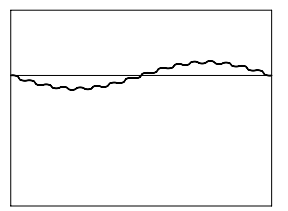

$t=2 \times 10^{-5}$

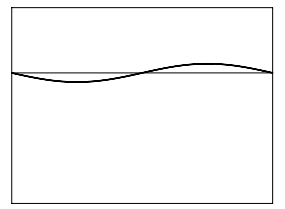

$t=4 \times 10^{-3}$

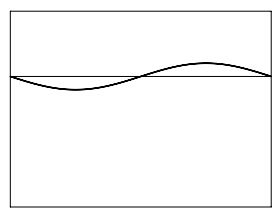

$t=3 \times 10^{-5}$

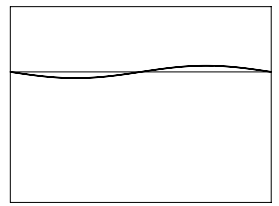

$t=8 \times 10^{-3}$

FIG. 7.1. Solutions for $f \equiv 0$ and $u_{0}(x)=1+0.1 \sin (\pi x)+0.3 \sin (16 \pi x)$ at various instants $t$. In all the plots, the $x$-axis ranges from -1 to 1 , and the $y$-axis ranges from 0 to 1.5 .

which results from the superposition of two frequencies. We compute the approximate solution with linear elements and parameters $h=1 / 128, \tau=10^{-6}$. This choice of discretization parameters is necessary to reflect the intrinsic time scale for this example. Figure 7.1 depicts the solution for different time instants and shows that high frequencies are rapidly damped, whereas the amplitude of low frequency waves decays very slowly. To quantify the difference in the time scales it is worth noting that the time elapsed between the first and the last plot of the first row of Figure 7.1 is $3 \times 10^{-5}$, whereas that of the second row is almost $10^{-2}$, a difference of three orders of magnitude. This is related to the fourth-order operator of surface diffusion.

Nonnegative perturbation. Let the perturbation be $\delta(x)=0.3 \delta_{0}(0.15 x)$, with

$$
\delta_{0}(x)=\min (1, \max (0,2-|x|)),
$$

which is nonnegative and rather singular for this fourth-order flow because of its kinks (see Figure 7.2). We compute the approximate solution with linear elements and parameters $h=1 / 128, \tau=10^{-6}$. Figure 7.2 displays the solution for different time instants and confirms the strong smoothing effect of surface diffusion alluded to before. Another important feature that can be visualized in Figure 7.2 is the lack of maximum principle for this equation: we start with a function $u_{0} \geq 1$ and, after the first time step, there are already points $x$ with $u(x)<1$. This is consistent with the fourth-order structure of the operator. It is also worth observing that the spectrum of $u_{0}$ is rather full due to the kinks and that high and low frequencies have drastically different decay rates.

Steep perturbation. This example shows that global in time existence may not be expected for a classical solution of (1.1), thereby revealing some limitations of the graph formulation. For $K=1+\frac{\sqrt{5}}{2}$, we take the perturbation $\delta(x)=0.3 \delta_{0}(0.15 x)$, with

$$
\delta_{0}(x)= \begin{cases}-K+(1+K)|x| & \text { if }|x|<1 \\ 2-|x| & \text { if } 1 \leq|x|<2 \\ 0 & \text { otherwise }\end{cases}
$$

$\delta$ is steep, and its mean value vanishes (see Figure 7.3). We compute the approximate solution with linear elements and parameters $h=1 / 128, \tau=10^{-7}$. The most impor- 


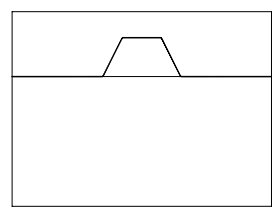

$t=0$

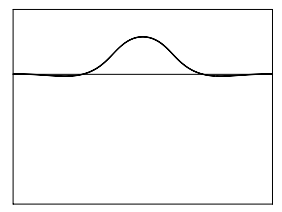

$t=2 \times 10^{-4}$

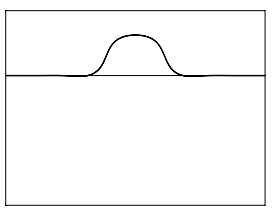

$t=1 \times 10^{-5}$

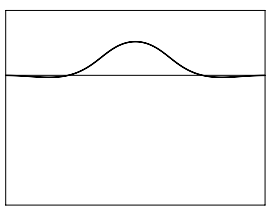

$t=4 \times 10^{-4}$

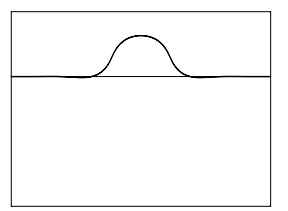

$t=2 \times 10^{-5}$

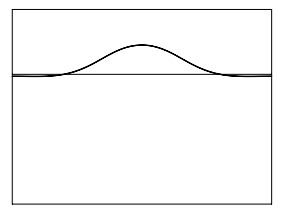

$t=8 \times 10^{-4}$

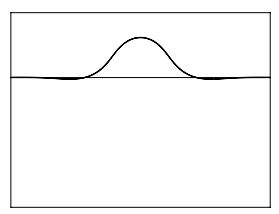

$t=1 \times 10^{-4}$

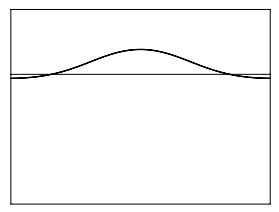

$t=16 \times 10^{-4}$

FIG. 7.2. Solutions for $f \equiv 0$ and $u_{0}(x)=1+\delta(x)$, with $\delta(x)$ a positive perturbation at various times $t$. In all the plots, the $x$-axis ranges from -1 to 1 , and the $y$-axis ranges from 0 to 1.5 .

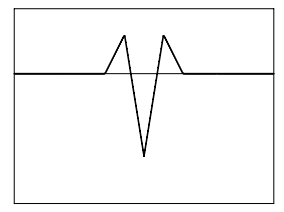

$t=0$

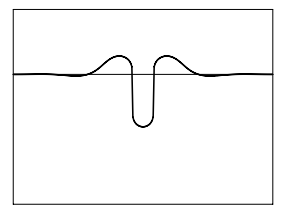

$t=4.8 \times 10^{-5}$

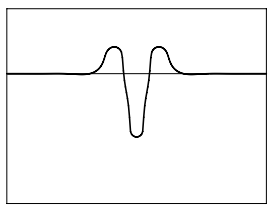

$t=8 \times 10^{-6}$

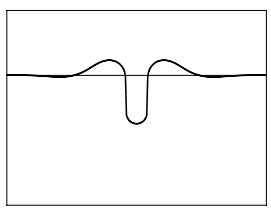

$t=9.6 \times 10^{-5}$

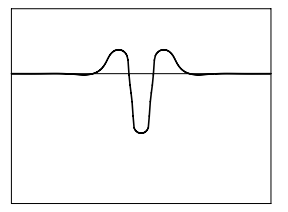

$t=16 \times 10^{-6}$

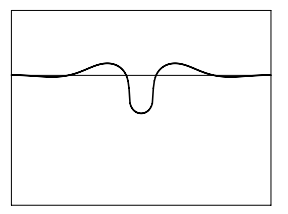

$t=19.2 \times 10^{-5}$

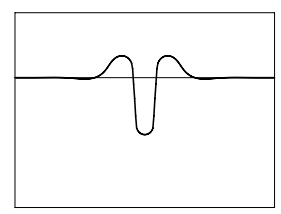

$t=24 \times 10^{-6}$

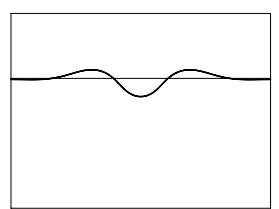

$t=38.4 \times 10^{-5}$

FIG. 7.3. Solutions for $f \equiv 0$ and $u_{0}(x)=1+\delta(x)$ at various times $t$, with a steep perturbation $\delta(x)$. In all the plots, the $x$-axis ranges from -1 to 1 , and the $y$-axis ranges from 0 to 1.5 .

tant features of $\delta$ are its steep slope, together with a big jump of the first derivative around $x=0$. As can be seen in Figure 7.3, the slope seems to become vertical around $t=4.8 \times 10^{-5}$, which indicates that the classical solution might cease to exist in finite time; in contrast, the discrete solution exists globally in time (see section 3). We stress that the lack of smoothness of $u_{0}$ plays a secondary role since starting with the (smooth) solution $u(t)$ for some small $t>0$ would yield the same evolution.

To investigate the formation of singularities in finite time, we use the parametric formulation of $[2,3]$ with the same initial data; for more examples and details about the discretization for parametric surfaces, we refer the reader to $[2,3]$. Since the parametric formulation works for closed curves and surfaces, we thus embed the graph of $u_{0}$ into a closed curve (see Figure 7.4, top left). For the time scale of Figure 7.3, the effect of this extension is negligible. Figure 7.4 displays a sequence of solutions obtained for the same eight time instants as in Figure 7.3. We see that the parametric evolution by surface diffusion tends to form a mushroom starting with this initial condition. Therefore, we conclude that the continuous solution will cease to be the graph of a function in finite time; i.e., the exact solution to the graph formulation of 


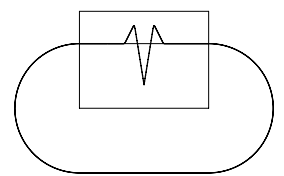

$t=0$

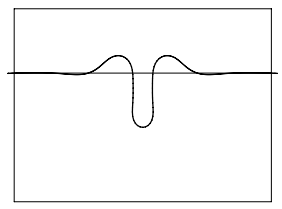

$t=4.8 \times 10^{-5}$

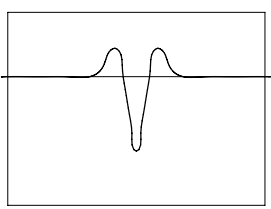

$t=8 \times 10^{-6}$

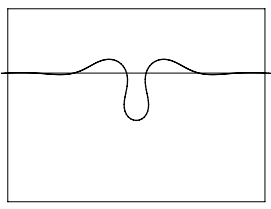

$t=9.6 \times 10^{-5}$

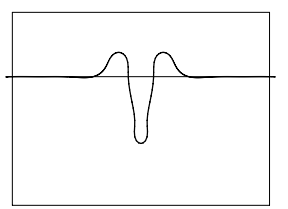

$t=16 \times 10^{-6}$

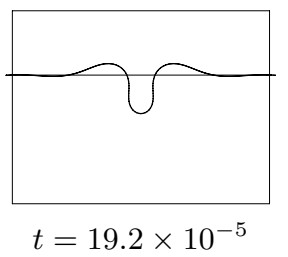

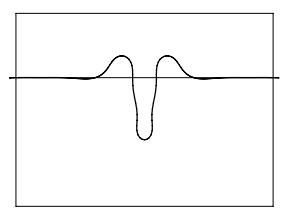

$t=24 \times 10^{-6}$

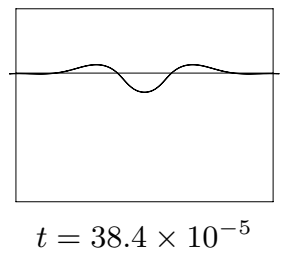

FIG. 7.4. Solutions obtained with a discretization for parametric curves from $[2,3]$ at the same times as in Figure 7.3. In all the plots, the rectangles in thin lines are $[-1,1] \times[0,1.5]$.

surface diffusion exists only locally in time for certain initial conditions. To assess the range of validity of the graph formulation, namely, to be able to detect blow-up, time and space adaptivity might be relevant. It is worth noticing the striking similarity of the solutions obtained with both methods. Even though the parametric solution develops a mushroom at $t=9.6 \times 10^{-5}$, and thus the solution to the graph formulation is questionable thereon, they still exhibit an excellent quantitative agreement for $t>9.6 \times 10^{-5}$ (compare the last two plots of Figures 7.4 and 7.3).

7.4. Smoothing effect in two dimensions: Case $\boldsymbol{f} \equiv \mathbf{0}$. In this section we present experimental results in $\Omega=(-1,1) \times(-1,1)$ concerning the behavior of the discrete solution when $f \equiv 0$ and $u_{0}(x)=1+\delta(x)$ is a perturbation of the solution $u \equiv 1$.

Positive perturbation. We consider a positive perturbation as depicted in Figure 7.5 and compute the approximate solution with linear elements and parameters $h=1 / 16$, $\tau=10^{-6}$. Figure 7.5 displays the solution for different time instants. We observe, as in the one-dimensional case, a strong smoothing effect much faster for high frequencies than for low frequencies, as well as the solution becoming less than 1 (lack of maximum principle).

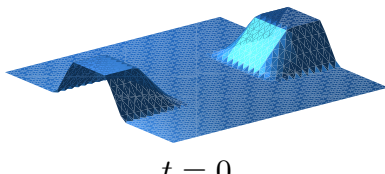

$t=0$

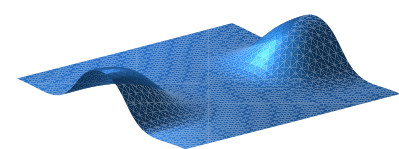

$t=1 \times 10^{-4}$

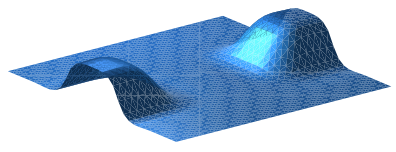

$t=1 \times 10^{-5}$

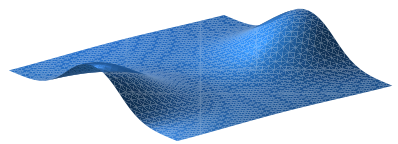

$t=5 \times 10^{-4}$

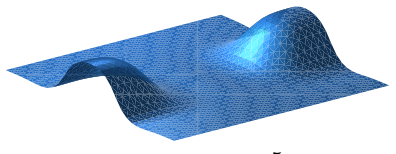

$t=5 \times 10^{-5}$

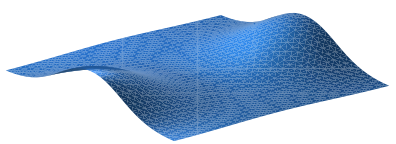

$t=10 \times 10^{-4}$

FIG. 7.5. Solutions for $f \equiv 0$ and $u_{0}(x)=1+\delta(x)$ at various time instants, with $\delta(x)$ a positive perturbation touching the periodic boundary. 
7.5. Crack formation in one dimension: Case $f=-C / u$. We study here the effect of a prescribed forcing of the form $f=-C / u$, which is motivated by the following stationary situation in one dimension and corresponding linearized stability analysis. The nonlinear evolution undergoes two distinct regimes: coarsening and crack formation. We define the latter as the instance when the height $u$ becomes zero at one or more points.

Equilibrium shape of deformable solids. Following [4], we consider a twodimensional thin solid occupying the domain $\{(x, y):-1 \leq x \leq 1,0 \leq y \leq u(x)\}$ and undergoing a plastic deformation due to competition of elastic effects and surface tension with volume constraint $\int_{-1}^{1} u=2$. The solid is to adjust its shape in order to minimize the following energy:

$$
\mathcal{I}(u, v, \lambda):=\int_{-1}^{1} \sqrt{1+\left|u_{x}\right|^{2}}+\frac{1}{2} \int_{-1}^{1} u\left|v_{x}\right|^{2}-\lambda\left(\int_{-1}^{1} u-2\right),
$$

where $u(x)$ describes the free surface of the film, $v(x)$ is the displacement of the solid, and $\lambda$ is a Lagrange multiplier associated with the volume constraint. Hence, the first term in (7.4) corresponds to surface tension, whereas the second one is the elastic energy, provided that the displacement $v$ solely depends on the horizontal variable $x$. Upon variational differentiation with respect to $u, v$, and $\lambda$, the Euler-Lagrange equations turn out to be

$$
-\left(\frac{u_{x}}{\sqrt{1+\left|u_{x}\right|^{2}}}\right)_{x}+\frac{1}{2}\left|v_{x}\right|^{2}-\lambda=0, \quad\left(u v_{x}\right)_{x}=0, \quad \int_{-1}^{1} u=2 .
$$

This immediately yields $v_{x}=\frac{C}{u}$, whence the equation for $u$ reads

$$
-\left(\frac{u_{x}}{\sqrt{1+\left|u_{x}\right|^{2}}}\right)_{x}+\frac{C}{u^{2}}-\lambda=0 .
$$

Linearized stability analysis. Since $u \equiv 1$ is a solution of (1.1), then a perturbation $w$ of $u$ evolves for a short time according to the linearized PDE around $u$ :

$$
\partial_{t} w=-\Delta\left(\Delta w+f^{\prime}(u) w\right),
$$

where $f(u)=-C / u^{\gamma}$ from the previous discussion, with $\gamma>0$. Taking an ansatz $w=e^{\mu t} e^{i \pi k x}$ periodic in $(-1,1)$, we obtain the spectral relation

$$
\mu=-(\pi k)^{4}+C \gamma(\pi k)^{2} .
$$

This implies that $\mu>0$, provided that $(\pi k)^{2}<C \gamma$, whence low frequency perturbations grow and the rest decay for a short time (linear regime).

In the simulations below, we make the simplest choice $\gamma=1$ and take $C=50$. Our goal is to explore the long-time behavior of (1.1) not predicted by (7.5) (nonlinear regime). We discretize the nonlinear forcing term $f(u)$ explicitly, namely, $f_{n+1}=$ $-I_{h}\left(C / u_{h}^{n}\right)$, and use linear finite elements with parameters $h=1 / 128, \tau=10^{-5}$.

Superposition of sines. We consider the sinusoidal perturbation of (7.1). Figure 7.6 displays the solution at different time instants and shows that high frequencies are rapidly damped, whereas the low frequencies slowly lead to a crack formation. This is consistent with the linearized stability analysis (7.5), according to which the frequency $k=1$ is the only unstable mode. 


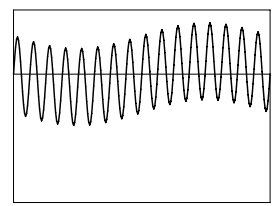

$t=0$

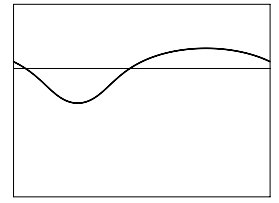

$t=1.8 \times 10^{-3}$

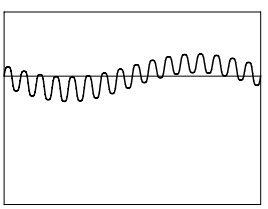

$t=3 \times 10^{-5}$

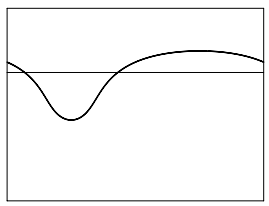

$t=2.2 \times 10^{-3}$

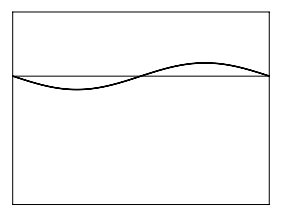

$t=1 \times 10^{-4}$

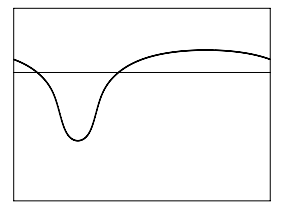

$t=2.5 \times 10^{-3}$

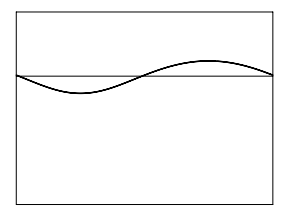

$t=6 \times 10^{-4}$

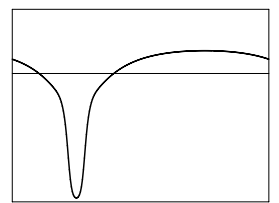

$t=2.66 \times 10^{-3}$

FIG. 7.6. Solutions for $f=-50 / u$ and $u_{0}(x)=1+0.1 \sin (\pi x)+0.3 \sin (16 \pi x)$ at various time instants. In all the plots, the $x$-axis ranges from -1 to 1 , and the $y$-axis ranges from 0 to 1.5 .

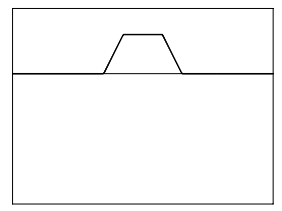

$t=0$

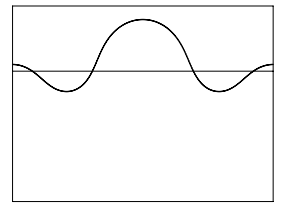

$t=2 \times 10^{-3}$

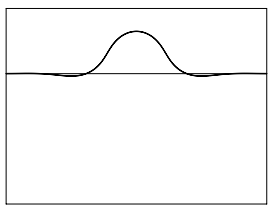

$t=1 \times 10^{-4}$

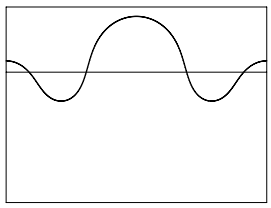

$t=2.5 \times 10^{-3}$

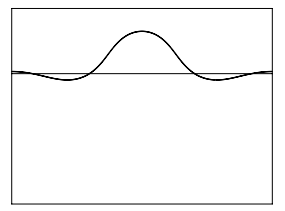

$t=5 \times 10^{-4}$

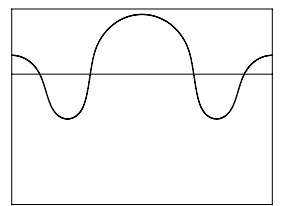

$t=3 \times 10^{-3}$

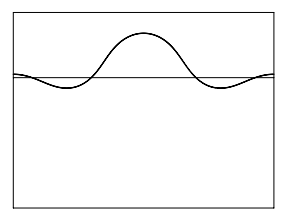

$t=1 \times 10^{-3}$

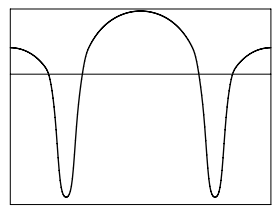

$t=3.5 \times 10^{-3}$

FIG. 7.7. Solutions for $f=-50 / u$ and $u_{0}(x)=1+\delta(x)$ at various time instants, with $\delta(x)$ the positive perturbation of (7.2). In all the plots, the $x$-axis ranges from -1 to 1 , and the $y$-axis ranges from 0 to 1.5 .

Positive perturbation. We consider the perturbation $\delta$ of (7.2) and display the results in Figure 7.7, which shows an evolution towards crack formation in finite time.

Small perturbation. We consider a perturbation $\delta(x)=0.1 \delta_{0}(0.02 x)$, with $\delta_{0}$ given in (7.3). Simulations are depicted in Figure 7.8, which shows that by $t=2 \times 10^{-5}$ the solution is smoothed out. It seems that we have reached a constant equilibrium for a relatively long time $t \cong 7.5 \times 10^{-3}$ (metastable state). Then an instability grows, and a fracture starts to form. The latter develops rather fast.

In order to shed light on the actual evolution during the transition between the fast smoothing of the perturbation and the crack development, we show in Figure 7.9 the solution at some time instants between $2 \times 10^{-5}$ and $7.5 \times 10^{-3}$, with the $y$-axis ranging between 0.998 and 1.001. Even though $u(t)$ looks constant to the eye in Figure 7.8 for $t$ in this interval, a magnification of the $y$-axis shows that this is not the case: some long waves survive the smoothing effect, and at some point their amplitudes start to increase. 


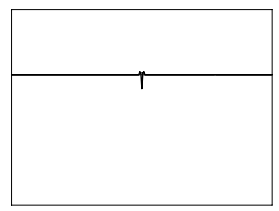

$t=0$

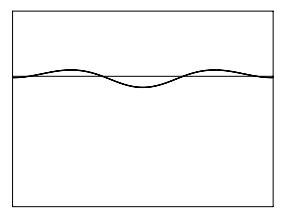

$t=1.25 \times 10^{-2}$

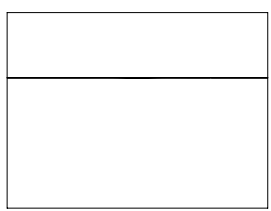

$t=2 \times 10^{-5}$

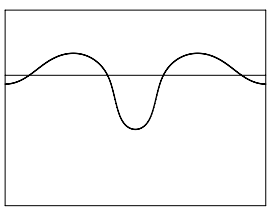

$t=1.5 \times 10^{-2}$

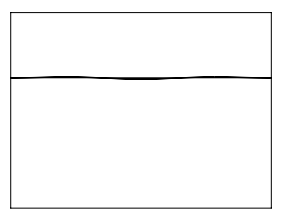

$t=7.5 \times 10^{-3}$

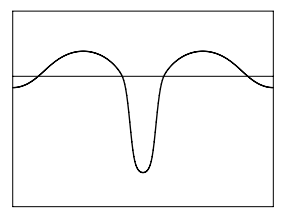

$t=1.53 \times 10^{-2}$

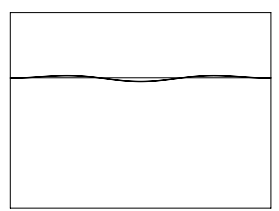

$t=1 \times 10^{-2}$

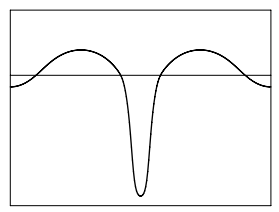

$t=1.533 \times 10^{-2}$

FIG. 7.8. Solutions for $f=-50 / u$ and $u_{0}(x)=1+\delta(x)$ at various time instants, with $\delta(x) a$ small Lipschitz perturbation. In all the plots, the $x$-axis ranges from -1 to 1 , and the $y$-axis ranges from 0 to 1.5 .

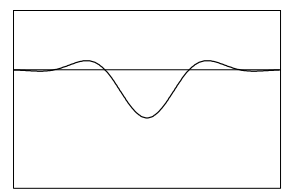

$t=2 \times 10^{-4}$

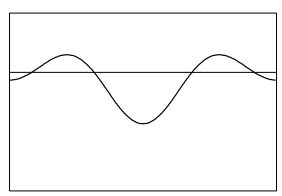

$t=1 \times 10^{-3}$

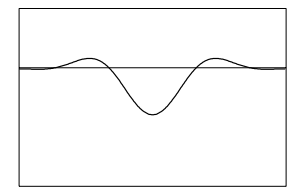

$t=2.5 \times 10^{-4}$

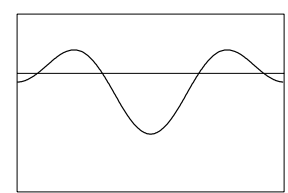

$t=1.5 \times 10^{-3}$

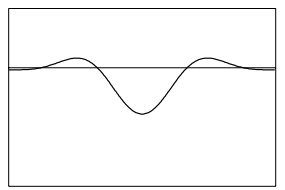

$t=3 \times 10^{-4}$

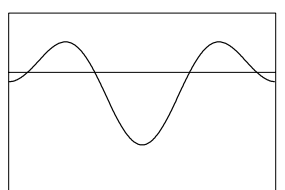

$t=2 \times 10^{-3}$
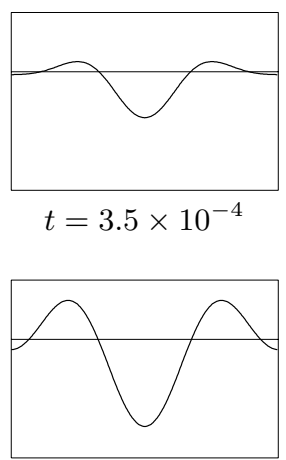

$t=2.5 \times 10^{-3}$

FIG. 7.9. Solutions for $f=-50 / u$ and $u_{0}(x)=1+\delta(x)$ at various time instants between $t=2 \times 10^{-5}$ and $t=7.5 \times 10^{-3}$, with the small perturbation of Figure 7.8 . In all the plots, the $x$-axis ranges from -1 to 1 , and the $y$-axis ranges from 0.998 to 1.001 .

Figure 7.10 displays the Fourier modes of $u(t)$ at times $t=0,10^{-5}, 10^{-2}, 3 \times 10^{-2}$. We observe that all the modes except the first two decrease immediately, whereas the first two modes increase. This is consistent with the prediction (7.5) of linearized stability because $k^{2} \pi^{2}<50$ implies $k \leq 2$.

Other simulations, also with forcing $f=-50 / u$, do not corroborate this apparent consistency with the linearized stability analysis. We observe that, for a fixed high frequency, the solution either develops a crack or tends to the steady solution $u=1$, depending on the size of the perturbation; for instance, if $u_{0}(x)=1+\alpha \sin (4 \pi x)$, then a crack forms for $\alpha \geq 0.2375$, thus violating the prediction $k^{2} \pi^{2}<50$ of (7.5). On the other hand, for a low frequency, the solution always develops a crack regardless of the magnitude of perturbation; for instance, if $u_{0}(x)=1+\alpha \sin (\pi x)$, then a crack forms for all $\alpha \in[0.001,0.5]$ tested. These simulations will be reported elsewhere. We also refer the reader to $[7,9]$, where simulations under the assumption of axial symmetry, but without forcing, are performed and singularities are observed as well, which do not conform to the linearized stability analysis either. 


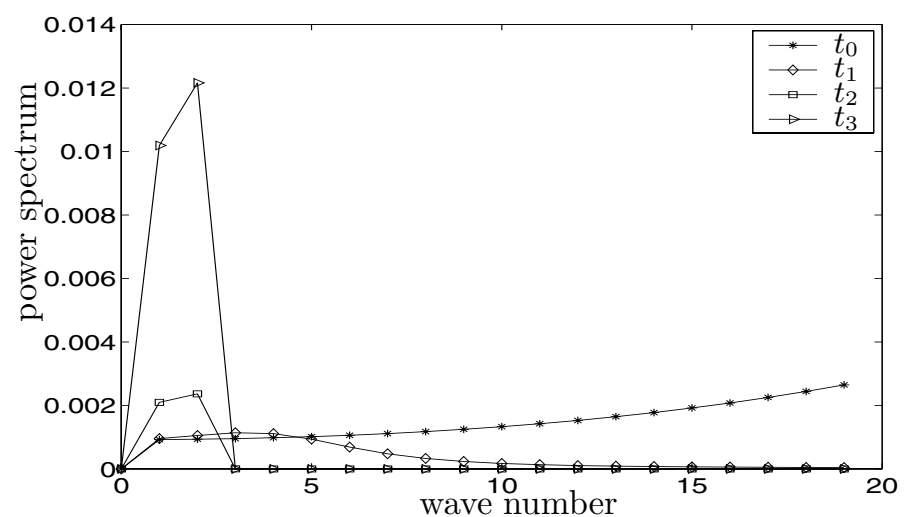

FIG. 7.10. Power spectrum for the solutions with $f=-50 / u$ and $u_{0}(x)=1+\delta(x)$, with the perturbation $\delta$ of Figure 7.8. The time instants are $t_{0}=0, t_{1}=10^{-5}, t_{2}=10^{-2}$, and $t_{3}=3 \times 10^{-2}$.

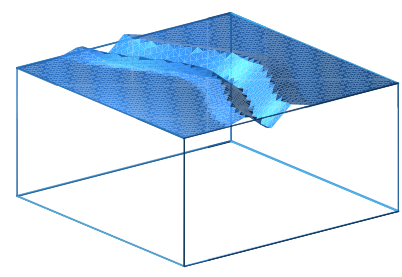

$t=0$

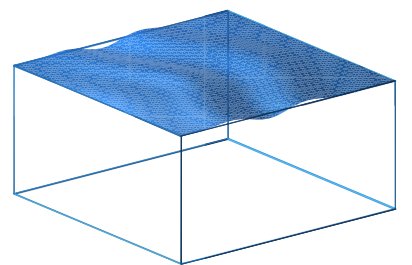

$t=1 \times 10^{-4}$

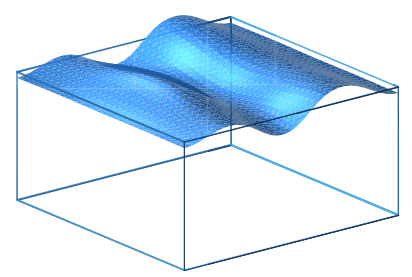

$t=5 \times 10^{-3}$

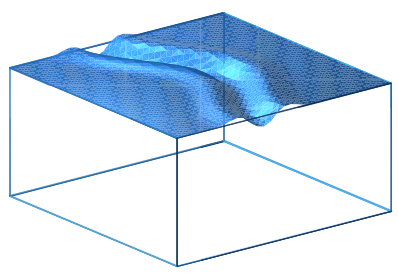

$t=5 \times 10^{-6}$

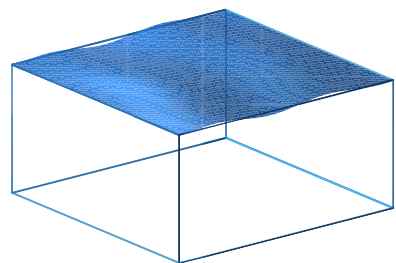

$t=1 \times 10^{-3}$

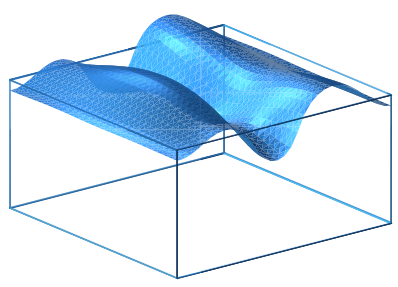

$t=6 \times 10^{-3}$

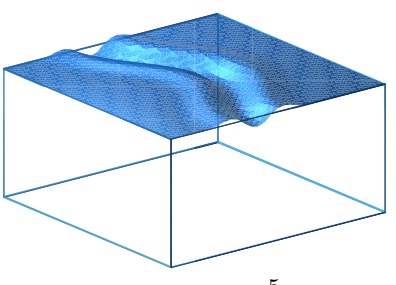

$t=1 \times 10^{-5}$

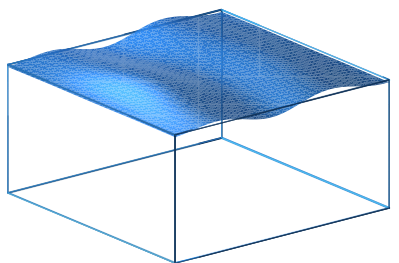

$t=3 \times 10^{-3}$

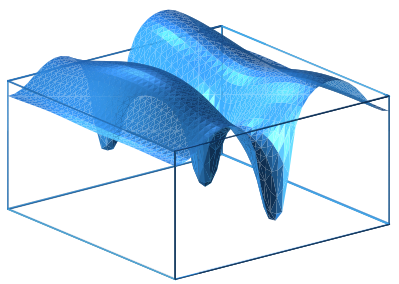

$t=6.5 \times 10^{-3}$

FIG. 7.11. Solutions for $f=-50 / u$ and $u_{0}(x)=1+\delta(x)$ at various time instants, with $\delta(x) a$ small perturbation across $y=\cos x$.

7.6. Crack formation in two dimensions: Case $f=-C / u$. We conclude this section with the evolution of two-dimensional surfaces immersed in $\mathbb{R}^{3}$. We consider again the initial surface to be $u_{0}=1+\delta$, where $\delta$ is a perturbation similar to that of Figure 7.3. First, we choose such $\delta$ across the periodic curve $y=\cos x$ (see Figure 7.11) and, finally, across the circle $x^{2}+y^{2}=1 / 4$ centered at the ori- 

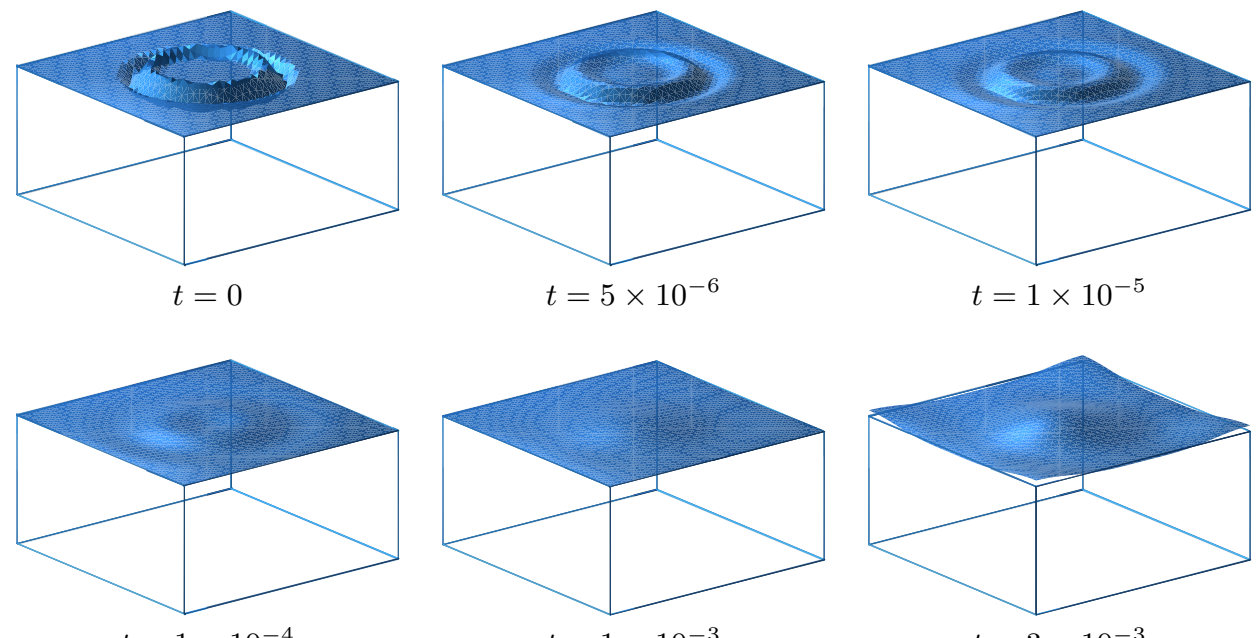

$t=1 \times 10^{-4}$

$$
t=1 \times 10^{-3}
$$

$$
t=3 \times 10^{-3}
$$
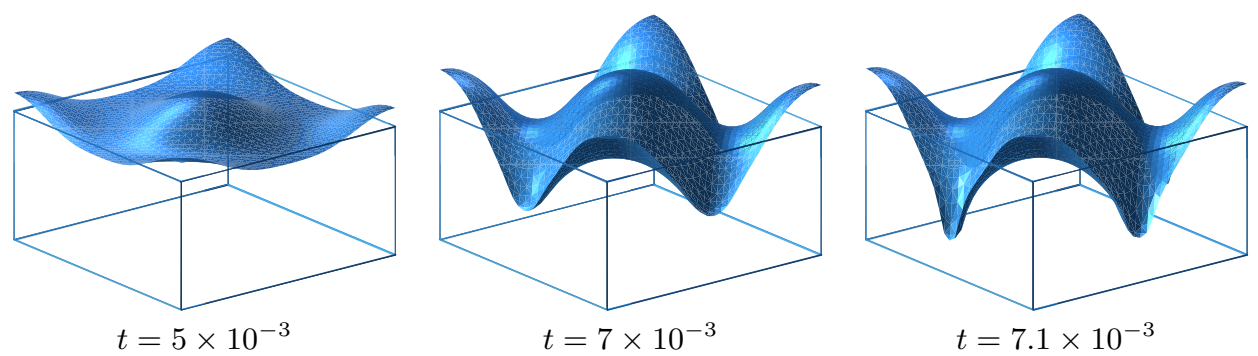

FIG. 7.12. Solutions for $f=-50 / u$ and $u_{0}(x)=1+\delta(x)$ at various time instants, with $\delta(x)$ a small perturbation across $x^{2}+y^{2}=1 / 4$.

gin (see Figure 7.12). We compute with linear elements and parameters $h=1 / 16$, $\tau=10^{-6}$.

We observe first a smoothing effect followed by crack formation. The latter seems to occur at isolated points rather than at lines, as illustrated in Figures 7.11 and 7.12. This happens even for one-dimensional profiles in two dimensions: point singularities seem to be preferred by this evolution.

Acknowledgments. We would like to thank J. Colin and M. Grinfeld, who originated our interest in surface diffusion, and F. Haußer for discovering (and fixing) a bug in our code.

\section{REFERENCES}

[1] R. J. Asaro and W. A. Tiller, Surface morphology development during stress corrosion cracking: Part I: Via surface diffusion, Metall. Trans., 3 (1972), pp. 1789-1796.

[2] E. Bänsch, P. Morin, And R. H. Nochetto, Finite element methods for surface diffusion, in Free Boundary Problems, Internat. Ser. Numer. Math. 147, P. Colli, C. Verdi, and A. Visintin, eds., Birkhäuser, Basel, 2003, pp. 53-63.

[3] E. Bänsch, P. Morin, And R. H. Nochetto, A Finite Element Method for Surface Diffusion: The Parametric Case, preprint.

[4] E. Bonnetier, R. FAlk, And M. Grinfeld, Analysis of a one-dimensional variational model of the equilibrium shape of a deformable crystal, M2AN Math. Model. Numer. Anal., 33 (1999), pp. 573-591. 
[5] S. C. Brenner And L. R. Scott, The Mathematical Theory of Finite Element Methods, Springer-Verlag, New York, 2002.

[6] J. Cahn And J. TAYlor, Surface motion by surface diffusion, Acta Metall. Mater., 42 (1994), pp. 1045-1063.

[7] B. Coleman, R. Falk, And M. Moakher, Space-time finite element methods for surface diffusion with applications to the theory of the stability of cylinders, SIAM J. Sci. Comput., 17 (1996), pp. 1434-1448.

[8] K. DeCKelnick AND G. DZIUK, Error estimates for a semi-implicit fully discrete finite element scheme for the mean curvature flow of graphs, Interfaces Free Bound., 2 (2000), pp. 341359.

[9] K. Deckelnick, G. Dziuk, And C. M. Elliott, Error analysis of a semidiscrete numerical scheme for diffusion in axially symmetric surfaces, SIAM J. Numer. Anal., 41 (2003), pp. 2161-2179.

[10] G. DZIUK, Numerical schemes for the mean curvature flow of graphs, in Variations of Domain and Free-Boundary Problems in Solid Mechanics, Solid Mech. Appl. 66, Kluwer Academic Publishers, Dordrecht, The Netherlands, 1999, pp. 63-70.

[11] C. M. Elliott and S. Maier-PaApe, Losing a graph with surface diffusion, Hokkaido Math. J., 30 (2001), pp. 297-305.

[12] J. Escher, U. F. Mayer, And G. Simonett, The surface diffusion flow for immersed hypersurfaces, SIAM J. Math. Anal., 29 (1998), pp. 1419-1433.

[13] M. A. Grinfeld, Thermodynamic Methods in the Theory of Heterogeneous Systems, Longman, New York, 1991.

[14] C. Herring, Surface tension as a motivation for sintering, in The Physics of Powder Metallurgy, W. E. Kingston, ed., McGraw-Hill, New York, 1951, pp. 143-179.

[15] W. W. Mullins, Theory of thermal grooving, J. Appl. Phys., 28 (1957), pp. 333-339.

[16] A. Schmidt and K. G. Siebert, ALBERT: An Adaptive Hierarchical Finite Element Toolbox, Documentation, Preprint 06/2000, Universität Freiburg, Freiburg, Germany, p. 244.

[17] A. Schmidt and K. G. Siebert, ALBERT - Software for Scientific Computations and Applications, Acta Math. Univ. Comenian. (N.S.), 70 (2001), pp. 105-122.

[18] B. J. Spencer, S. H. Davis, and P. W. Voorhees, Morphological instability in epitaxiallystrained dislocation-free solid films: Nonlinear evolution, Phys. Rev. B, 47 (1993), pp. 97609777.

[19] B. J. Spencer AND D. I. MeIRon, Nonlinear evolution of the stress-driven morphological instability in a two-dimensional semi-infinite solid, Acta Metall. Mater., 42 (1994), pp. 36293641.

[20] D. J. SRolovitz, On the stability of surfaces of stressed solids, Acta Metall., 37, (1989), pp. $621-625$. 